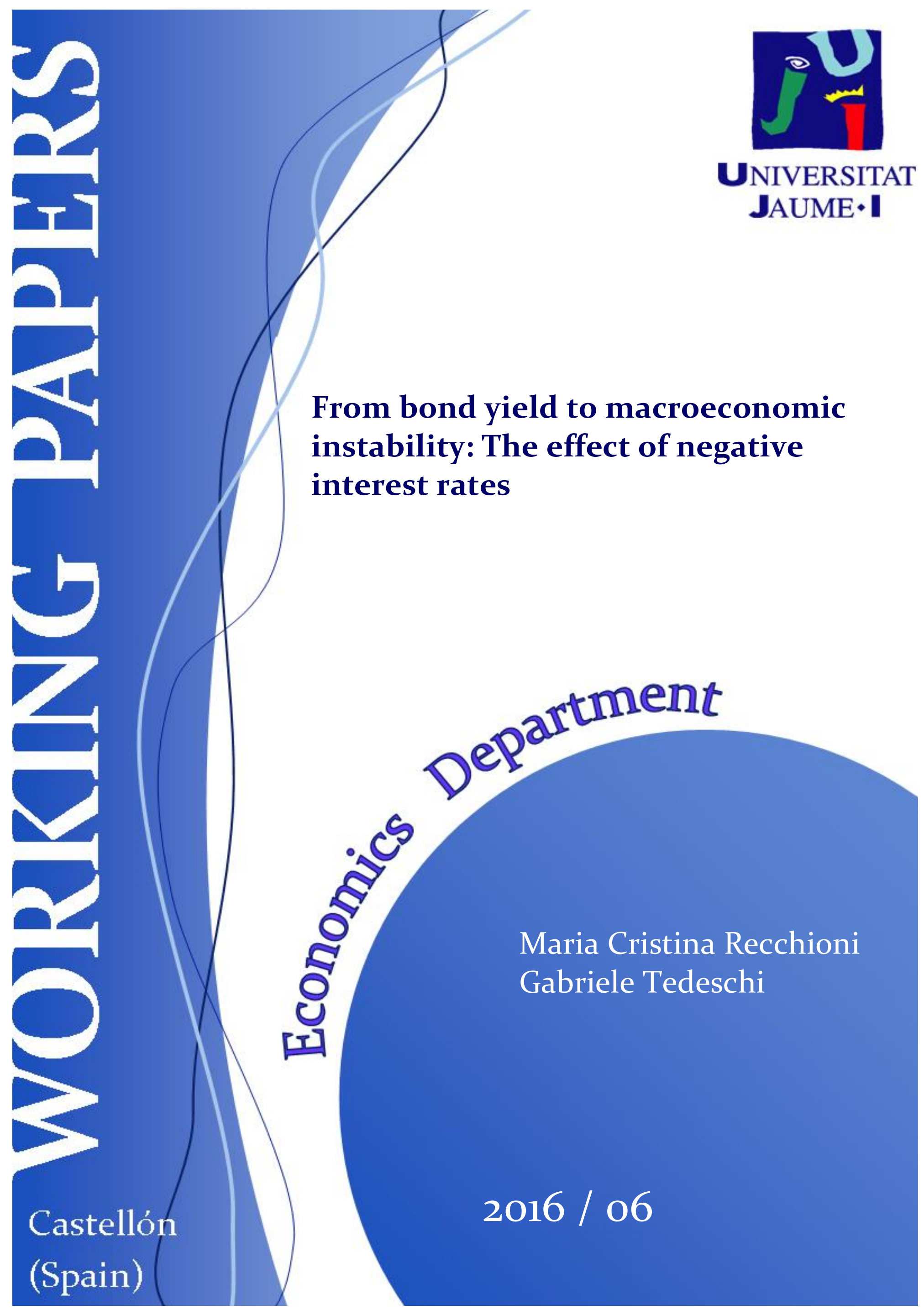




\title{
From bond yield to macroeconomic instability: The effect of negative interest rates
}

\author{
Maria Cristina Recchioni \\ Università Politecnica delle Marche \\ Department of Managment \\ m.c.recchioni@univpm.it
}

\author{
Gabriele Tedeschi \\ Universitat Jaume I \\ Department of Economics \\ gabriele.tedeschi@gmail.com
}

2016 / 06

\begin{abstract}
We present a hybrid Heston model with a local stochastic volatility to describe government bond yield dynamics. The model is analytically tractable and, therefore, can be efficiently estimated using the maximum likelihood approach. Twofold is the model contribution. First, it captures changes in the yield volatility and predict future yield values of Germany, French, Italy and Spain. The result is an early-warning indicator which anticipates phases of instability characterizing the time series investigated. Then, the model describes convergence/divergence phenomena among European government bond yields and explores the countries' reactions to a common monetary policy described through the EONIA interbank rate.
\end{abstract}

Keywords: Stochastic volatility model, Kolmogorov backward equation, maximum likelihood function, government bond yield forecasting.

JEL classification: C13, C32, G12, G17, E58. 


\title{
From bond yield to macroeconomic instability: the effect of negative interest rates
}

\author{
Maria Cristina Recchioni ${ }^{a}$, Gabriele Tedeschi $^{b}$ \\ a) Universitá Politecnica delle Marche, Ancona, Italy and b) Universitat Jaume I, Castellón, Spain \\ E-mail: m.c.recchioni@univpm.it; gabriele.tedeschi@gmail.com
}

April 28, 2016

\begin{abstract}
We present a hybrid Heston model with a local stochastic volatility to describe government bond yield dynamics. The model is analytically tractable and, therefore, can be efficiently estimated using the maximum likelihood approach. Twofold is the model contribution. First, it captures changes in the yield volatility and predict future yield values of Germany, French, Italy and Spain. The result is an earlywarning indicator which anticipates phases of instability characterizing the time series investigated. Then, the model describes convergence/divergence phenomena among European government bond yields and explores the countries' reactions to a common monetary policy described through the EONIA interbank rate.
\end{abstract}

JEL classification: C13, C32, G12, G17, E58

Keywords: Stochastic volatility model, Kolmogorov backward equation, maximum likelihood function, government bond yield forecasting.

\section{Introduction}

The financial and economic crisis that started in 2007 is a clear symbol of the materialization and propagation of systemic risk.

Systemic risk and the potential ensuing contagion refer to a situation whereby the instability in a given country, market or institution is transmitted to one or more countries, markets or institutions. On the one hand, the strong interaction at the micro and meso level generated the well-known knock-on effect, which culminated in the demise of Lehman Brothers. On the other hand, the same interdependence at the macro level has played a key role in exacerbating the sovereign debt problems in the Euro zone. 
As a consequence, macro and financial economists and market participants have all attempted to build reliable models to describe and anticipate systemic risk. Although the resulting models are very different in form and fit, they all incorporate the interactions as a key element in generating crisis and contagion. A significant part of the literature focuses on the analysis of government bond yields. These important financial instruments, in fact, reflect the interaction phenomena from different angles. First, they incorporate information on the relationship among countries and their mutual interdependence in government debt. In this regard, the literature has studied the convergence (divergence) of government bond yields in Europe and especially among the Euro Area countries ${ }^{1}$. In many papers this is done by attributing an important role to the fiscal/monetary policies in causing such convergence (divergence) (see, for instance, Rault and Afonso 2011; Afonso and Strauch 2007; Attinasi et al. 2011; Mesters et al. 2014; Manganelli and Wolswijk 2009; Sgherri and Zoli 2009). Second, the yield term structure provides important information about how to evaluate a country with respect to its development over time. In this regard, the above mentioned interactions become dynamic and describe phenomena in the short, medium and long term (see, for instance, Diebold and Li 2006; Ehrmann et al. 2011; Von Hagen et al. 2011; Trolle and Schwarz 2009).

In this paper we are interested in analyzing both the first line of research, interactions among countries, and the second line, the countries' development over time via yield curves. Specifically, we propose a simple analytically tractable stochastic volatility model in continuous time which captures the yield dynamics in the Eurozone. The model is based upon an important assumption: the interest rate volatility is stochastic and common across the different yields investigated. The stochasticity of the interest rate volatility is a well-known stylized fact about interest rate (see, for example, Trolle and Schwarz 2009). The fact that this volatility is common ${ }^{2}$ is due to the strong political and economical ties among the countries analyzed. To sum up the model describes the dynamics of $n$ yields which depend, each in a different way, on a common stochastic volatility described by a mean reverting process.

We deduce an integral representation formula for the transition/marginal conditional density function of the process as well as an explicit expression for its moments. Furthermore, we propose an expansion of the marginal conditional density function in powers of the volatility of volatility, and derive the first two terms of this expansion. These two

\footnotetext{
${ }^{1} \mathrm{~A}$ part of the research has focused on the determinants of yield spread between European countries and other States (see Nickel et al. 2011; Giannone et al. 2011).

${ }^{2}$ We also generalized the model using two volatilities: one common to all yields and responsible for the yield volatility changes, the other depends on the yield maturity and is responsible for the yield cross section. This generalized model is still analytically tractable. Its ability to simultaneously describe government bond yields with different maturities and representing different countries will be the object of future research.
} 
terms are elementary functions and are used to obtain a closed form formula approximation for the transition probability function and the cumulative distribution function. This simple perturbation approach, applicable to several stochastic volatility models, allows us to cope with the curse of dimensionality which arises when an efficient calibration of the model is necessary. This in turn permits an efficient estimation of model parameters, yielding reliable time series of these parameters, whose analysis provides useful insights into market behavior.

Two classes of models "compete" in being able to reproduce the yield curve. The first are macroeconomic models which study how the market/government expectation of inflation and future real economic activity determine the yields. This group of models often use a reduced-form term structure where bond yields are expressed using three factors: "level", "steepness', and "curvature". Starting from the the pioneering Nelson and Siegel (1987) model and its re-interpretation by Diebold and Li (2006), several reduced-form term structure models have been developed over the last ten years. These models have proven to be quite successful at capturing and forecasting the cross-sectional properties of bond yields (see Diebold et al 2006b; Diebold et al. 2008; Diebold and Rudebusch 2013; Hautsch and Ou 2012; Chen and Tsang 2013; Mesters et al. 2014). Moreover, they have shown that level, slope and curvature factors also capture systematic risk. The second class are financial models which study derivatives pricing and portfolio risk management. Foremost among these are the popular affine arbitrage-free term structure models (see Dong et al. 2002; Chiarella and Kwon 2003; Cheredito et al. 2007; Collin-Dufresne et al. 2009; Andersen and Benzoni 2010). This class of models focuses on fitting the term structure at a point in time to ensure good forecasts of derivatives and portfolio risk. However, in recent years, these models have employed factors capable of capturing the stochastic volatility of the interest rates. Thanks to this, these models have been able to describe and predict the bond yield term structure ${ }^{3}$ (see Dai and Singleton 2002, Duffee 2002; Collin-Dufresne et al. 2009; Trolle and Schwartz 2009).

Despite the impressive theoretical advances of the yield curve in macroeconomics and financial economics, a large gap still exists between these two classes of models. Surprisingly, little attention has been paid to analyzing the potential bidirectional feedback between the yield curve to macroeconomic dynamics. This is particularly true for financial modeling that does not consider the impact that macroeconomic policies may have on the yield curve. This paper begins to bridge this gap by formulating and estimating a yield

\footnotetext{
${ }^{3}$ There are interesting contributions which combine the two groups of models. For instance, Coroneo et al. (2011) show that a reduced-form term structure model is compatible with arbitrage-freeness. Instead, Christensen et al. 2011, 2014 and Mesters et al. 2014 introduce stochastic volatilities in reduced-form term structure models.
} 
model that integrates financial and macroeconomic factors. To this end, we introduce an affine model, which is a hybrid Heston model with a local stochastic volatility, to describe government bond yield dynamics (see Homeschu 2011; Trolle and Schwartz 2009). We estimate our stochastic volatility model on German, French, Italian and Spanish bond yields and on the EONIA interbank rate from 29 March, 2004 to 3 April, 2014. The selected countries are chosen as being representative of different geographical areas of the Eurozone while the time period considered is relevant due to the presence of different economic phases. Furthermore, the introduction of the EONIA interest rate ${ }^{4}$ allows us to analyze the effects of the monetary policy not only with respect to the investigated countries but also with respect to economic phases.

Due to its simplicity and analytical tractability, the model is able to capture changes in yield volatility and predict future yield values. Its descriptive and predictive abilities are verified not only on fixed-maturity bonds, but also on bonds with different maturities. The reason for this good performance of the model rests on two important features. First, the derivation of a closed form solution for the cumulative distribution function and explicit formulas for the moments allow us to efficiently estimate the model parameters via the maximum likelihood approach. Second, the assumption of a common stochastic volatility governing the Eurozone allows us, on the one hand, to simplify the analytical treatment and, on the other hand, to understand the current interactions among the countries of this zone.

The model's good performance in reproducing the yield curve encourages us to further study the properties of the estimated parameters. The empirical and mathematical results suggest a strong correlation between the estimated volatility parameters and the instability in the government bond yields. Thus, starting from the analysis of these parameters we are able to build an early warning indicator for significant instabilities. The proposed indicator identifies three bubbles that anticipate the three episodes of instability characterizing our time series: the sub-prime mortgage, the collapse of Lehman and the sovereign debt crisis. We also investigate the potential of this indicator on U.S. data (treasury bills). The results obtained confirm that the calibrated model is able to capture the peculiarity of the markets analyzed.

Having successfully validated our estimate for in-sample fitting and out-of-sample forecasting, we illustrate two other abilities of the model. First, its ability to describe the relations among European countries and, second, its ability to foresee their reactions to economic policies or shocks that occur in Eurozone.

In order to address the relationships among the countries investigated we analyze the

\footnotetext{
${ }^{4}$ The EONIA interest rate is often seen as a proxy of European monetary policy (see Giannone et al. 2011; Mesters et al. 2014; Lucas et al. 2014).
} 
dynamics of the specific country volatility which is one of the key model parameters. This parameter allows us to understand, not only phenomena of convergence (divergence) among countries, but also their macroeconomic (in)stability. The results of the empirical analysis indicate a strong co-movement between France and Germany on the one hand, and Italy and Spain on the other. Moreover, as shown in other empirical studies (see Mesters et al. 2014) the country volatilities behave differently during the period investigated. In fact, the volatility of all countries dramatically increases during the financial crisis (2007-2008) while only in Italy and Spain does it remain elevated due to the sovereign debt crisis (2011-2012).

In order to address the impact of monetary policies on countries, we analyze their reactions to changes in the EONIA rate. Our empirical analysis reveals that the BCE expansionary monetary policies have a strong impact in mitigating the instability of the countries investigated between 2009 and 2011. However, these monetary policies do not seem equally incisive in alleviating the sovereign debt crisis which negatively affects the two Mediterranean countries.

It is worth noting that the empirical results obtained with our financial model confirm and reinforce some important findings already highlighted in some recent macroeconomic models (see Mesters et al. 2014; Afonso and Martins 2012). The fact that financial and macroeconomic models generate very similar results not only confirms the soundness of the results, but also the utility of merging the two methodologies. In fact, they should not be seen in competition, but rather, in Popperian falsifiability.

The rest of the paper is organized as follows. In Section 2 we present the stochastic volatility model. In Section 3 we introduce the maximum likelihood approach to estimate the model parameters. We also investigate the robustness of the procedure on simulated data. In Section 4 we present the numerical experiments on government bond yields. Finally, in Section 5 we draw conclusions.

\section{The multivariate stochastic volatility model}

In this section we introduce the stochastic volatility model for yields/interest rates. Let $\mathbb{R}^{+}$be the set of positive real numbers, $n$ a positive integer and $\mathbb{R}^{n}$ the $n$-dimensional real Euclidean space.

We denote with $x_{i, t}, i=1,2, \ldots, n$, the stochastic yields/interest rates and with $v_{t}$ their variance at time $t>0$. We assume that the real vector valued stochastic process 
$\left(x_{i, t}, v_{t}\right)$ is described by the following system of stochastic differential equations:

$$
\begin{aligned}
& d x_{i, t}=\mu_{i} d t-\frac{\tilde{m}}{2} \sigma_{i}^{2} v_{t} d t+\sigma_{i} \sqrt{v_{t}} d W_{i, t}, t>0, i=1,2, \ldots, n, \tilde{m}=0,1, \\
& d v_{t}=\chi\left(\theta-v_{t}\right) d t+\varepsilon \sqrt{v_{t}} d Q_{t}, \quad t>0
\end{aligned}
$$

with initial conditions:

$$
\begin{aligned}
x_{i, 0} & =\tilde{x}_{i, 0}, \quad i=1,2, \ldots, n \\
v_{0} & =\tilde{v}_{0}
\end{aligned}
$$

where $\tilde{x}_{i, 0}, i=1,2, \ldots, n$, and $\tilde{v}_{0}$ are random variables concentrated in a point with probability one ${ }^{5}$. The parameters $\chi, \theta, \varepsilon, \sigma_{i}$ in Eqs. (1)-(2) are suitable real constants satisfying the following conditions:

$$
\begin{aligned}
\epsilon, \chi, \theta, \sigma_{i} & \geq 0, \\
\frac{2 \chi \theta}{\epsilon^{2}} & \geq 1,
\end{aligned}
$$

while $\mu_{i}$ is a drift term that, for simplicity, is assumed to be constant in time ${ }^{6}$. Moreover, $W_{i, t}$ and $Q_{t}$ are standard Wiener processes such that $W_{i, 0}=0, Q_{0}=0$ while $d W_{i, t}$ and $d Q_{t}$ denote their stochastic differentials. Equation (1) depends on the integer $\tilde{m}=0 ; 1$ and negative values of $x_{i, t}$ are allowed. This improves the model's ability to mimic historical data. Condition (6) guarantees a positive variance, $v_{t}$, for any $t>0$ with probability one given that $v_{0}$ is positive with probability one.

Furthermore, we assume that these stochastic differentials satisfy the following conditions:

$$
\begin{aligned}
& E\left(d W_{i, t} d Q_{t}\right)=\rho_{v, i} d t, \quad i=1,2, \ldots, n, \\
& E\left(d W_{i, t} d W_{j, t}\right)=\rho_{i, j} d t, \quad i \neq j, \quad i, j=1,2, \ldots, n, \\
& E\left(d W_{i, t} d W_{i, t}\right)=d t, \quad i=1,2, \ldots, n, \\
& E\left(d Q_{t} d Q_{t}\right)=d t,
\end{aligned}
$$

where $E(\cdot)$ denotes the expected value of $\cdot$, and $\rho_{v, i}, \rho_{i, j} \in(-1,1)$ are constant correlation coefficients.

To sum up, the stochastic volatility model (1)-(2) describes the log-price in the Heston model when $\tilde{m}=1$ and $n=1$, while for $n>1$ and $\tilde{m}=1$ it can be interpreted as a particular case of the model proposed by Trolle and Schwartz (2009).

\footnotetext{
${ }^{5}$ For the sake of simplicity, we use $x_{0}$ and $v_{0}$ instead of their initial values $\tilde{x}_{0}$ and $\tilde{v}_{0}$.

${ }^{6}$ The theoretical results proposed hold under the assumption that $\mu_{i}, i=1,2, \ldots, n$ are integrable functions of time. In the empirical analysis (forecasting exercise) we choose $\mu_{i}$ to be time dependent as in Diebold and Li (2006).
} 
Our interpretation of the parameters of the stochastic volatility model (1)-(2) strictly depends on two empirical applications:

a) bonds of different countries with fix-maturity;

b) bonds of one country with several maturities.

In application (a) the process $x_{i, t}$ describes the yield of the $i-t h$ country and the variance $v_{t}$, measures the volatility of government sovereign bond yields in a given zone. In the application (b) $x_{i, t}$ describes the yield with the $i-t h$ maturity and $v_{t}$ is the variance which drives the entire yield term structure. In addition, the parameter $\sigma_{i}$, which is set equal to one in the Heston model, describes the specific volatility of the $i-t h$ country in application (a) and of the $i-t h$ maturity in application (b). As in Heston 1993, the parameters $\theta, \chi$, $\epsilon$ identify the long term mean, the speed of mean reversion and the volatility of volatility ( vol of vol) respectively.

We proceed with the analytical treatment of the stochastic volatility model (1)-(2) defining the transition probability density function, $p_{f}$, of process $\left(\underline{x}_{t}, v_{t}\right), t>0$. Specifically, the arguments of $p_{f}$ include "past" and "future" variables: $(\underline{x}, v, t)=\left(x_{1}, x_{2}, . ., x_{n}, v, t\right)$ are the "past" variables and $\left(\underline{x}^{\prime}, v^{\prime}, t^{\prime}\right)=\left(x_{1}^{\prime}, . ., x_{n}^{\prime}, v^{\prime}, t^{\prime}\right)$ the "future" ones, since $t<t^{\prime}$.

Let us denote with $M$ the marginal conditional probability density defined by:

$$
M\left(\underline{x}, v, t, \underline{x}^{\prime}, t^{\prime}\right)=\int_{0}^{+\infty} p_{f}\left(\underline{x}, v, t, \underline{x}^{\prime}, v^{\prime}, t^{\prime}\right) d v^{\prime}, t^{\prime}>t .
$$

Following Duffie, Pan and Singleton (2000) we derive the following integral representation formula for $M$ :

$$
\begin{gathered}
M\left(\underline{x}, v, t, \underline{x}^{\prime}, t^{\prime}\right)=\frac{1}{(2 \pi)^{n}} \int_{\mathbb{R}^{n}}\left\{e^{-\imath \underline{k}^{T}\left(\underline{x}-\underline{x}^{\prime}+\left(t^{\prime}-t\right) \underline{\mu}\right)} e^{-\frac{v}{2}(a(\underline{k})-\imath c(\underline{k})) \varphi\left(t^{\prime}-t, \underline{k}\right) .}\right. \\
\left.e^{-\frac{2 \chi \theta\left(t^{\prime}-t\right)}{\epsilon^{2}}(\nu(\underline{k})+\zeta(\underline{k}))} e^{-\frac{2 \chi \theta}{\epsilon^{2}} \ln \left(1+\frac{(\nu(\underline{k})+\zeta(k))}{2 \zeta(\underline{k})}\left(e^{-2 \zeta(\underline{k})\left(t^{\prime}-t\right)}-1\right)\right)}\right\} d \underline{k}, \\
t<t^{\prime}, \underline{x}, \underline{x}^{\prime} \in \mathbb{R}^{n}, v>0,
\end{gathered}
$$

where $\imath$ is the imaginary unit, the superscript $T$ denotes the transpose and $\underline{\mu}=\left(\mu_{1}, \mu_{2}, \ldots, \mu_{n}\right)$. Formula (12) provides the marginal density $M$ as a Fourier integral where $\underline{k}$ is conjugate variable of the yield variable.

The first quantity on the right hand side of Eq. (12) is:

$$
a(\underline{k})=\underline{k}^{T} \Gamma \underline{k},
$$


where $\Gamma$ is the matrix given by:

$$
\Gamma_{i, j}=\left\{\begin{array}{ll}
\sigma_{i} \rho_{i, j} \sigma_{j} & i \neq j \\
\sigma_{i}^{2} & i=j
\end{array} \quad i, j=1,2, \ldots, n .\right.
$$

The second quantity is:

$$
c(\underline{k})=\tilde{m} \underline{k}^{T} \underline{\sigma},
$$

with $\underline{\sigma}=\left(\sigma_{1}^{2}, \sigma_{2}^{2}, \ldots, \sigma_{n}^{2}\right) \in \mathbb{R}^{n}$. Finally, the quantities $\varphi, \nu, \zeta$ are:

$$
\begin{gathered}
\varphi(s, \underline{k})=\frac{1-e^{-2 s \zeta(\underline{k})}}{(\nu(\underline{k})+\zeta(\underline{k})) e^{-2 s \zeta(\underline{k})}+(\zeta(\underline{k})-\nu(\underline{k}))}, \\
\nu(\underline{k})=-\frac{1}{2}(\chi+\imath \epsilon b(\underline{k})), \\
\zeta(\underline{k})=\left[\nu(\underline{k})^{2}+\frac{\epsilon^{2}}{4}(a(\underline{k})-\imath c(\underline{k}))\right]^{1 / 2},
\end{gathered}
$$

where $b$ is:

$$
b(\underline{k})=\underline{k}^{T} \underline{\rho}_{v},
$$

and $\underline{\rho}_{v}$ is:

$$
\underline{\rho}_{v}=\left(\sigma_{1} \rho_{v, 1}, \sigma_{2} \rho_{v, 2}, \ldots, \sigma_{n} \rho_{v, n}\right) .
$$

The reader can find the derivation of the formula for the marginal conditional probability density function in Appendix A.

Formula (12) involves a $n$ dimensional Fourier integral. Given the special form of the integrand function, it can be computed using an "ad hoc" Monte Carlo method. However, we avoid the curse of dimensionality by approximating $M$ using the first two terms of its series expansion in powers of the vol of vol, $\epsilon$, with base point $\epsilon=0$.

The expansion of the marginal conditional density function in powers of vol of vol, $\epsilon$, is the main mathematical contribution of this paper. It allows us to develop a calibration procedure capable of detecting "calm" and "turbulent" financial periods. The basic idea is that in a "calm" financial period, the yield distribution is Gaussian (i.e. $\epsilon=0$ ) with time dependent mean reverting volatility. In contrast, in a "turbulent" period, the distribution moves abruptly towards something different from a Gaussian distribution (i.e. $\epsilon>0$ ) and simultaneously large values of the initial stochastic volatility, $v_{0}$, are estimated. However, determining these changes in distribution using the analytical expression of the marginal density is challenging due to the curse of dimensionality. This is the reason for an expansion of the analytical marginal conditional probability density function in powers of the vol of vol, $\epsilon$. The marginal conditional probability density function is therefore expressed as a normal density function plus a correction term given by a closed-form expression. 
The expansion method proposed here can obviously be applied to any stochastic volatility model where the probability density function can be explicitly computed when vol of vol, $\epsilon$, is set to zero. In detail, we assume that the following expansion for $M$ holds:

$$
M\left(\underline{x}, v, t, \underline{x}^{\prime}, t^{\prime}\right)=\sum_{j=1}^{+\infty} \epsilon^{j} M_{j}\left(s, \underline{x}, \underline{x}^{\prime}, v\right), \quad s=t^{\prime}-t>0 .
$$

The reader can find the derivation of the analytical formulas of the first two terms, $M_{0}$, $M_{1}$, of the expansion in Appendix B:

$$
\begin{gathered}
M_{0}\left(s, \underline{x}, \underline{x}^{\prime}, v\right)=\frac{1}{(2 \pi)^{n / 2}} \frac{e^{-\frac{1}{2 f_{1}(s, v)}\left(\underline{x}-\underline{x^{\prime}}+s \underline{\mu}-\frac{\tilde{m}}{2} f_{1}(s, v) \underline{\sigma}\right)^{T} \Gamma^{-1}\left(\underline{x}-\underline{x^{\prime}}+s \underline{\mu}-\frac{\tilde{m}}{2} f_{1}(s, v) \underline{\sigma}\right)}}{\sqrt{\left(f_{1}(s, v)\right)^{n} \operatorname{det} \Gamma}}, \\
M_{1}\left(s, \underline{x}, \underline{x}^{\prime}, v\right)=-\frac{f_{2}(s, v)}{\chi \frac{\partial}{\partial v} f_{1}(s, v)} \frac{\partial}{\partial v} \sum_{i=1}^{n} \sigma_{i} \rho_{v, i} \frac{\partial}{\partial x_{i}^{\prime}} M_{0}\left(s, \underline{x}, \underline{x}^{\prime}, v\right),
\end{gathered}
$$

with

$$
f_{1}(s, v)=\theta s+(v-\theta)\left(\frac{1-e^{-\chi s}}{\chi}\right)
$$

and

$$
f_{2}(s, v)=(2 \theta-v)\left(s-\frac{\left(1-e^{-\chi s}\right)}{\chi}\right), s>0, v>0 .
$$

Assuming $\Gamma$ to be a positive definite matrix, formulas (22)-(23) are well defined since the function $f_{1}$ is positive for any $s>0$ and $v>0$

It is worth noting that the zero-th order term $M_{0}$ of the marginal conditional probability density function is a Gaussian density function and $M_{1}$ is defined by using the gradient of $M_{0}$. The simple analytical expression of $M_{0}$ and $M_{1}$ (see Eqs. (22)-(23)) allows us to obtain an efficient approximation of the cumulative distribution function (see Appendix B):

$$
\begin{gathered}
\operatorname{Pr}\left(\underline{x}_{t=s} \leq \underline{x}^{*}\left|\underline{x}_{t=0}=\underline{x}_{0}\right| v_{t=0}=v_{0}\right)= \\
\prod_{j=1}^{n} N\left(y_{j}^{*}\left(s, \underline{x}_{0}, v_{0}\right)\right)\left(1-\epsilon \frac{f_{2}\left(s, v_{0}\right)}{\chi \sqrt{f_{1}\left(s, v_{0}\right)} \frac{\partial}{\partial v} f_{1}\left(s, v_{0}\right)} V\left(s, v_{0}, \underline{y}^{*}\right)\right)
\end{gathered}
$$

where $N(x)=\frac{1}{\sqrt{2 \pi}} \int_{-\infty}^{x} e^{-\xi^{2} / 2} d \xi$ and the functions $V$ and $y_{q}^{*}, q=1,2, \ldots, n$ are given by:

$$
\begin{aligned}
& V\left(s, v_{0}, \underline{y}^{*}\right)= \\
& \left(-\frac{\frac{\partial}{\partial v} f_{1}(s, v)}{f_{1}(s, v)}+\sum_{q=1}^{n} \frac{\frac{\partial}{\partial v} N\left(y_{q}^{*}\left(s, \underline{x}_{0}, v\right)\right)}{N\left(y_{q}^{*}\left(s, \underline{x}_{0}, v\right)\right)}\right) \cdot \sum_{i=1}^{n} \sigma_{i} \rho_{v, i} \sum_{q=1}^{n} \frac{\Gamma_{q, i}^{-1 / 2}}{N\left(y_{q}^{*}\left(s, \underline{x}^{0}, v\right)\right)} \frac{e^{-\left(y_{q}^{*}\right)^{2} / 2}}{\sqrt{2 \pi}} \\
& +\sum_{i=1}^{n} \sigma_{i} \rho_{v, i} \sum_{q=1}^{n} \frac{\Gamma_{q, i}^{-1 / 2}}{N\left(y_{q}^{*}\left(s, \underline{x}^{0}, v\right)\right)} \frac{e^{-\left(y_{q}^{*}\right)^{2} / 2}}{\sqrt{2 \pi}}\left(-y_{q}^{*} \frac{\partial y_{q}^{*}}{\partial v}-\frac{\frac{\partial}{\partial v} N\left(y_{q}^{*}\left(s, \underline{x}_{0}, v\right)\right)}{N\left(y_{q}^{*}\left(s, \underline{x}_{0}, v\right)\right)}\right)
\end{aligned}
$$


and

$$
\underline{y}^{*}\left(s, \underline{x}_{0}, v\right)=\frac{1}{\sqrt{f_{1}(s, v)}} \Gamma^{-1 / 2}\left(\underline{x}^{*}-\underline{x}^{0}-s \underline{\mu}+f_{1}(s, v) \frac{\tilde{m}}{2} \underline{\sigma}\right) .
$$

Finally, we obtain the analytical expression of the first four conditional moments when $\tilde{m}=0$ and $\mu_{j}=0, j=1,2, \ldots, n$ (see formulas (99)-(101) in Appendix C) and of the first two moments in the general case (see formulas (102) and (103) in Appendix C).

\section{The estimation procedure}

In this section we use the maximum likelihood approach to estimate the parameters of the stochastic model (1)-(2). This model is parameterized by $3+3 n+n(n-1) / 2$ real quantities, that is the quantities: $\chi, \theta, \epsilon, \mu_{i}, \sigma_{i}, i=1,2, \ldots, n, \rho_{v, i}, i=1,2, \ldots, n, \rho_{i, j}$, $i=1,2, \ldots, n-1, j=i+1, i+2, \ldots, n$. Moreover, we consider the initial stochastic volatility $v_{0}$ as a parameter that must be determined. This is motivated by the fact that the initial volatility $v_{0}$ is not observable in the financial markets. Let us formulate the estimation problem by introducing the set of feasible parameters:

$$
\begin{gathered}
\mathcal{S}=\left\{\underline{\Theta} \in \mathbb{R}^{4+3 n+n(n-1) / 2}, \underline{\Theta}=\left(\epsilon, \theta, \chi, v_{0}, \mu_{i}, \sigma_{i}, \rho_{v, i}, \rho_{i, j}\right), \mid \epsilon, \chi, \theta, v_{0} \geq 0,\right. \\
\frac{2 \chi \theta}{\epsilon^{2}}>1, \sigma_{i}>0,-1<\rho_{v, i}<1, i=1,2, \ldots, n, \\
\left.-1<\rho_{i, j}<1, i=1,2, \ldots, n-1, j=i+1, i+2, \ldots, n\right\} .
\end{gathered}
$$

Let $m_{o b}$ be a positive integer, $\tilde{x}_{i, m}$ the $i$-th yield observed at $t=t_{m}, m=1,2, \ldots, m_{o b}$ and $\underline{\tilde{x}}_{m}=\left(\tilde{x}_{1, m}, \tilde{x}_{2, m}, \ldots, \tilde{x}_{n, m}\right)$. We choose $t_{m}<t_{m+1}, m=1,2, \ldots, m_{o b}$ where $t_{m_{o b}+1}$ denotes the current time.

We consider the (log-)likelihood function given by:

$$
\begin{gathered}
F(\underline{\Theta})=\frac{1}{\left(m_{o b}-1\right)} \sum_{m=1}^{m_{o b}-1} \ln M^{a}\left(t_{m+1}-t_{m}, \underline{\tilde{x}}_{m}, \underline{\tilde{x}}_{m+1}, \tilde{v}_{t_{m+1}} \mid \underline{\Theta}\right), \\
\underline{\Theta} \in \mathcal{M}, q=0,1, \ldots,
\end{gathered}
$$

where $M^{a}$ is as follows:

$$
M^{a}\left(s, \underline{x}, \underline{x}^{\prime}, v \mid \underline{\Theta}\right)=M_{0}\left(s, \underline{x}, \underline{x}^{\prime}, v\right)+\epsilon M_{1}\left(s, \underline{x}, \underline{x}^{\prime}, v\right), \quad \underline{\Theta} \in \mathcal{S},
$$

and $M_{j}$ are given in Eqs. (22), (23). We evaluate $v_{t}$ via the conditional expected value $\theta\left(1-e^{-\chi\left(t-t_{m}\right)}\right)+v_{t_{m}} e^{-\chi\left(t-t_{m}\right)}, t_{m} \leq t \leq t_{m+1}$, where $v_{t_{0}}=v_{0}$ and $v_{0}$ is the initial stochastic value which must be estimated (see Trolle et al. 2009, for a similar approach).

We solve the following estimation problem:

$$
\max _{\underline{\Theta} \in \mathcal{S}} F(\underline{\Theta}) .
$$


Problem (32) can be stated as follows:

given the observations $\left(\tilde{x}_{1, m}, \tilde{x}_{2, m}, \ldots, \tilde{x}_{n, m}\right)$ at time $t=t_{m}, m=1,2, \ldots, m_{o b}$, determine the vector $\underline{\Theta}^{*} \in \mathcal{S} \subset \mathbb{R}^{4+2 n+n(n-1) / 2}$ that makes these observations "more likely".

Problem (32) is solved via a variable metric steepest ascent method. Specifically, starting from an initial guess $\underline{\Theta}^{0} \in \mathcal{S}$, the iterative procedure updates the current approximation of the solution of (32) with a step in the direction of the gradient of the (log-)likelihood function (30) computed in a suitable metric (see Miglierina et al. 2008; Pacelli and Recchioni 2000). The estimation procedure can be summarized as follows:

1 set $k=0$ and initialize $\underline{\Theta}^{0}$;

2 evaluate $F\left(\underline{\Theta}^{k}\right)$, if $k>0$ and $\left|F\left(\underline{\Theta}^{k}\right)-F\left(\underline{\Theta}^{k-1}\right)\right|<\eta_{\max }\left|F\left(\underline{\Theta}^{k}\right)\right|$, where $|\cdot|$ denotes the absolute value of $\cdot$, go to step 7 ;

3 evaluate the gradient of the function $F\left(\underline{\Theta}^{k}\right)$;

4 perform the steepest ascent step evaluating $\underline{\Theta}^{k+1}=\underline{\Theta}^{k}+\eta_{k} D\left(\underline{\Theta}^{k}\right) \nabla F\left(\underline{\Theta}^{k}\right)$, where $D\left(\underline{\Theta}^{k}\right)$ is a diagonal matrix related to the use of the "variable metric" and $\eta_{k}$ is the length of the step in the direction $D\left(\underline{\Theta}^{k}\right) \nabla F\left(\underline{\Theta}^{k}\right)$ which guarantees that $F\left(\underline{\Theta}^{k}\right)$ is a non-decreasing function of $k$;

5 if $\left\|\underline{\Theta}^{k+1}-\underline{\Theta}^{k}\right\|<\eta_{\max }$, go to step 7 ;

6 set $k=k+1$, if $k<M_{\text {iter }}$ go to step 2 ;

7 take $\underline{\Theta}^{k+1}$ as the approximation of $\underline{\Theta}^{*}$ and stop.

The quantity $\eta_{\max }$ is a positive fixed tolerance value and $M_{i t e r}$ is the maximum number of iterations allowed in the optimization procedure.

The solution is determined by solving the same problem starting from several initial points. Specifically, we explore the feasible region $\mathcal{S}$ taking a set of random points belonging to $\mathcal{S}$ and evaluating the objective function at these points. The initial points of the iterative procedure are the points where the objective function attains its largest values.

We conclude the presentation of the estimation procedure by analyzing the performance of the approximation in Eq. (31). This is crucial because Eq. (31) is used in the formulation of the estimation problem (32). To this end, we set $n=4, \tilde{m}=0, \theta=0.1$, $\chi=2, \sigma_{v, j}=1, \rho_{v, j}=0.5, \mu_{j}=0, j=1,2, \ldots, n, \rho_{i, j}=0, i, j=1,2, \ldots, n, i \neq j$. Furthermore, given that $M_{a}\left(s, \underline{x}, \underline{x}^{\prime}, v_{0} \mid \underline{\Theta}\right)=M_{0}\left(s, \underline{x}, \underline{x}^{\prime}, v_{0}\right)+\epsilon M_{1}\left(s, \underline{x}, \underline{x}^{\prime}, v_{0}\right)$, we consider 
the following five values of $\epsilon, \epsilon_{1}=0, \epsilon_{2}=0.005, \epsilon_{3}=0.01, \epsilon_{4}=0.05, \epsilon_{5}=0.1$ and the following ten values of $v_{0}$ and $s, v_{0}=v_{0, j}=0.2 j, s=s_{j}=0.1 j, j=1,2, \ldots, 10$. Finally, we set $\underline{x}=(0.04,0.1861,0.0386,0.365)$ and $\underline{x}^{\prime}=\underline{x}(1+h)$ with $h=1$. These parameter values come from the empirical analysis on the real data (see Section 4).

The accuracy of the approximation $M_{a}$ to the marginal conditional density $M$ (see Eq. $(12))$ is measured as the average relative error, $E_{\epsilon}$ :

$$
E_{\epsilon}=\sum_{i=1}^{10} \sum_{j=1}^{10}\left|\frac{M\left(s_{i}, \underline{x}, \underline{x}^{\prime}, v_{0, j}\right)-\left(M_{0}\left(s_{i}, \underline{x}, \underline{x}^{\prime}, v_{0, j}\right)+\epsilon M_{1}\left(s_{i}, \underline{x}, \underline{x}^{\prime}, v_{0, j}\right)\right)}{M\left(s_{i}, \underline{x}, \underline{x}^{\prime}, v_{0, j}\right)}\right| .
$$

The integral, $M$, in Eq. (12) has been computed using an ad hoc Monte Carlo method

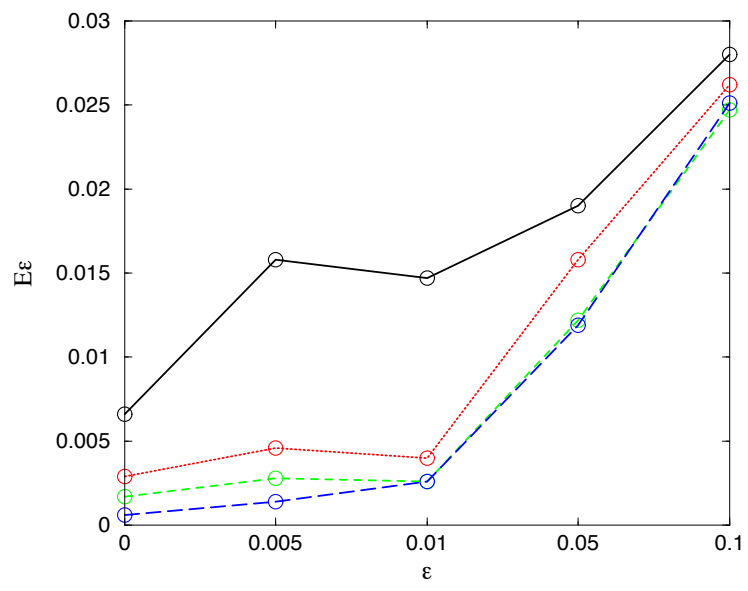

Figure 1: Average relative error, $E_{\epsilon}$, (see Eq. 33) as a function of $\epsilon$, for different Monte Carlo sample size, $N_{M C}$, equal to $10^{4}$ black solid line, $10^{5}$ red dotted line, $10^{6}$ green dashed line and $10^{7}$ blue long dashed line. Colors are available on the web site version.

with four different sample sizes $N_{M C}$ (i.e. $N_{M C}=10^{4}, N_{M C}=10^{5}, N_{M C}=10^{6}$ and $N_{M C}=10^{7}$ ).

Figure 1 shows the average relative error $E_{\epsilon}$ as a function of $\epsilon$ for each of the four Monte Carlo simulations. The four different sample sizes, each of which is represented by the lines in Fig.1, and the five different values of $\epsilon$ allow us to evaluate, on the one hand, the error of the Monte Carlo approximation and, on the other hand, the error of the expansion in $\epsilon$. Our experiment shows that increasing the Monte Carlo size the value of $E_{\epsilon}$ decreases significantly for $\epsilon \leq 0.01$ while it remains substantially unchanged for $\epsilon=0.05$ and $\epsilon=0.1$. This means that, for the largest values of $\epsilon$, the error is mainly due to the expansion approximation. However, for smaller values of $\epsilon$, the error, $E_{\epsilon}$, decreases as the sample size, $N_{M C}$, increases. In this circumstance, the error is mainly due to the Monte Carlo approximation. Finally, the long blue dashed line in Figure 1 shows that for $\epsilon \leq 0.01$ the first order approximation provides at least three correct significant digits 
while it provides only two significant correct digits for $\epsilon>0.01^{7}$.

As shown in the next section, the vol of vol, $\epsilon$, estimated from the real data is on average below $4 \%$ and thus we can expect the approximation, $M^{a}$, to work satisfactorily on the empirical studies too.

\subsection{The performance of the estimation procedure on simulated data}

We now investigate the performance of the estimation procedure and its ability to capture changes in model parameters.

We simulate the financial time series by integrating numerically Eqs. (1)-(2) through the explicit Euler discretization scheme with variable step-size less than $10^{-5}$. We simulate $n=4$ bond yield trajectories, $x_{i, t}$, and their stochastic variance, $v_{t}$, over a period of $T=8$ (years). Specifically, for each yield we simulate approximately three observations a day (i.e. about 800 observations a year) corresponding to a total of $N_{T}=6400$ observations. Each yield represents a specific country or maturity denoted by $C_{i}, i=1,2, \ldots, n$. We divide the simulated period in four time windows of the same length (1600 consecutive observations). We set the parameters of our benchmark model ${ }^{8}$ as follows: $\tilde{m}=0$, the vol of vol $\epsilon=0.01$, the long term mean $\theta=0.015$, the speed of mean reversion $\chi=0.05$. The specific yield volatility, $\sigma_{i}$ and the correlation coefficient between $C_{i}$ and $C_{j}, \rho_{i, j}$, are 0.7 and 0.5 respectively. The initial value of the yields is 0.2 (i.e. $x_{i, 0}=0.2, i=1,2, \ldots, 4$ ) and the drift terms, $\mu_{i}$, are set to zero. The correlation coefficient between the stochastic variance $v_{t}$ and the country/ maturity $C_{i}$ is equal to $\rho_{v, 1}=0.1, \rho_{v, 2}=0.1 \rho_{v, 3}=-0.1$, $\rho_{v, 4}=-0.1$. Finally, the initial stochastic volatility $v_{0}$ is equal to $v_{0}=0.0015$ in the first and third window (i.e. in years 0-2 and years 4-6 corresponding to 1-1600 and 3201-4008 observations) and $v_{0}=2.5$ in the second and fourth window (i.e. in years $2-4$ and years 6-8 corresponding to 1601-3200 and 4009-6400 observations). That is, in each time window the values of the model parameters are constant with the only exception of the initial stochastic volatility $v_{0}$.

We estimate the model parameters by using 400 consecutive observations which means solving estimation problem (32) sixteen times.

Our estimation problems requires sixteen initial starting points, $\underline{\Theta}^{0} \in \mathcal{S}$. They are generated using the spot volatility estimator by Mancino and Recchioni (2015) which is able to approximate the yield volatilities (i.e. $\left.\sigma_{j}^{2} v_{t}, j=1,2, \ldots, 4\right)$. By combining these ap-

\footnotetext{
${ }^{7}$ When the Monte Carlo sample size, $N_{M C}$, is equal to $10^{7}$ (blue long dashed line in Figure 1) the average relative errors, $E_{\epsilon}$, are $0.0006,0.0014,0.0026,0.0119$ and 0.0251 for each of the five considered values of $\epsilon$ respectively.

${ }^{8}$ The parameter values of the "benchmark" model come from the empirical analysis on the real data (see Section 4).
} 


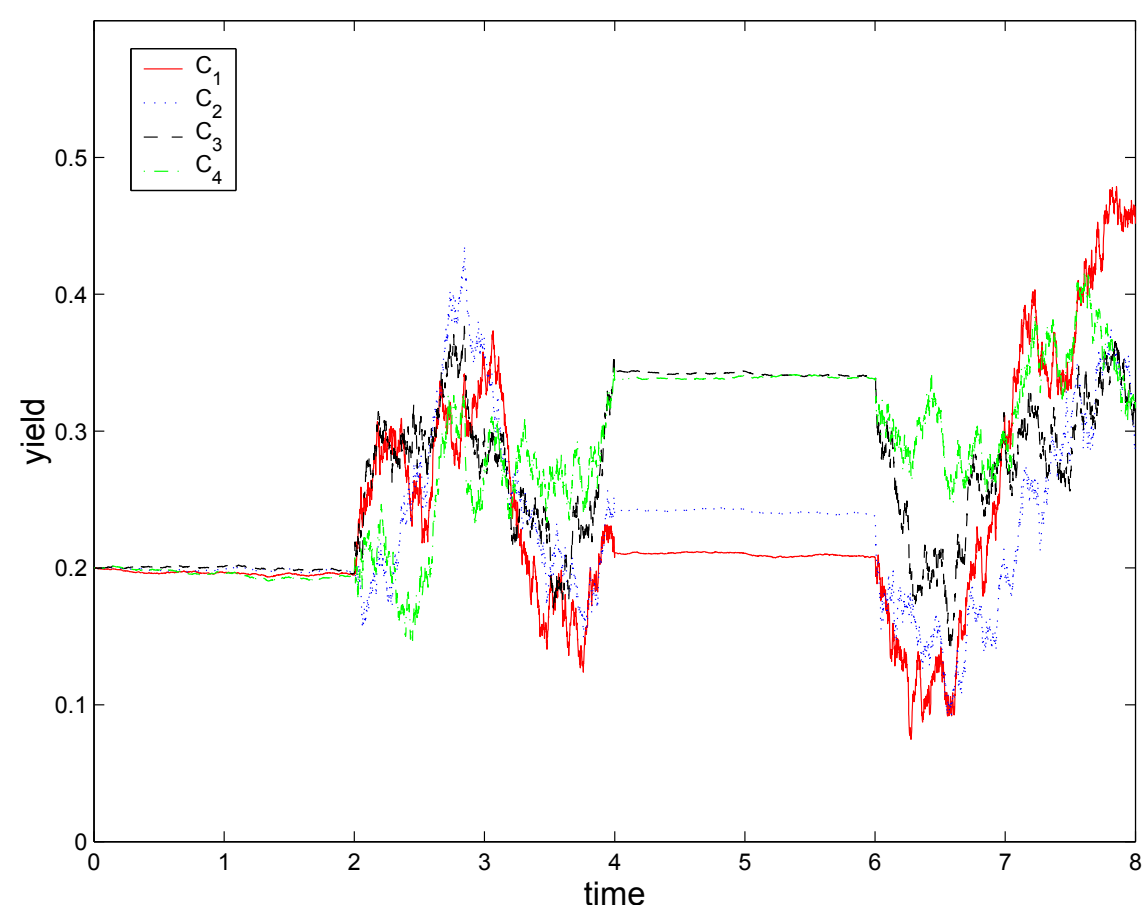

\begin{tabular}{cc}
\hline $\mathrm{q}$ & $\mathrm{v}_{0}(1.5 \mathrm{e}-3)$ \\
\hline 1 & $1.204081 \mathrm{e}-3$ \\
2 & $1.670453 \mathrm{e}-3$ \\
3 & $2.150294 \mathrm{e}-3$ \\
4 & $2.124346 \mathrm{e}-3$ \\
\hline $\mathrm{q}$ & $\mathrm{v}_{0}(2.5)$ \\
\hline 5 & 2.467920 \\
6 & 2.406428 \\
7 & 2.343765 \\
8 & 2.268779 \\
\hline $\mathrm{q}$ & $\mathrm{v}_{0}(1.5 \mathrm{e}-3)$ \\
\hline 9 & $1.059292 \mathrm{e}-3$ \\
10 & $1.333648 \mathrm{e}-3$ \\
11 & $1.662283 \mathrm{e}-3$ \\
12 & $1.914864 \mathrm{e}-3$ \\
\hline $\mathrm{q}$ & $\mathrm{v}_{0}(2.5)$ \\
\hline 13 & 2.470610 \\
14 & 2.420748 \\
15 & 2.371615 \\
16 & 2.309784 \\
\hline
\end{tabular}

Figure 2: Left panel: simulated yield time series as functions of time (expressed in years). Right panel: estimated values of the initial stochastic volatility $v_{0}$ obtained solving the sixteen calibration problems indexed by $q$ and "true" values of $v_{0}$ in brackets.

proximations with equations (1)-(2), we can provide proxies for the full set of the model parameters.

Figure 2 (left side) shows the simulated time series of each yield. We can observe that shocks in $v_{0}$ (years 2-4 and 6-8) generate abrupt changes of the yields. From a mathematical point of view it is quite obvious that an abrupt change in the value of the initial stochastic volatility, $v_{0}$, is able to generate strong fluctuations in simulated time series. However, this simple finding help us in interpreting the results coming from the empirical analysis proposed in Section 4, where strong fluctuations in the real time series can be predicted by strong variations in the estimated values of the initial stochastic volatility. In order to test the performance of our procedure in predicting strong yield fluctuations we must show our technique well reproduces the abrupt jumps in the parameter.

The performance is shown in the right hand side of Figure 2 where the estimated values of $v_{0}$ in the sixteen different time windows indexed by $q$ are displayed. The table confirms that the estimation procedure is able to capture the true values of the parameters and their changes over the entire time interval.

Table 1 shows the robustness of the estimation technique in replicating all the remaining parameters. We want to underline that the "true" values of these parameters are 
Table 1: "True" parameter values (first column); average values and standard deviations of the estimated parameters (second column); average relative errors of the estimated model parameters (third column). All the average values are computed by using the 16 estimated values of the parameters coming for the solution of the estimation problems indexed by $q$.

\begin{tabular}{ccc}
\hline Parameter $($ True $)$ & Ave. value $($ St. Dev.) & Ave. relative error \\
\hline$\epsilon(0.01)$ & $0.0101(0.0005)$ & 0.0397 \\
$\theta(0.015)$ & $0.0148(0.0004)$ & 0.0304 \\
$\chi(0.05)$ & $0.0494(0.0013)$ & 0.0242 \\
$\sigma_{1}(0.07)$ & $0.0691(0.0023)$ & 0.0281 \\
$\sigma_{2}(0.07)$ & $0.0709(0.0024)$ & 0.0269 \\
$\sigma_{3}(0.07)$ & $0.0699(0.0024)$ & 0.0248 \\
$\sigma_{4}(0.07)$ & $0.0699(0.0023)$ & 0.0265 \\
$\rho_{v, 1}(0.1)$ & $0.0911(0.0383)$ & 0.2696 \\
$\rho_{v, 2}(0.1)$ & $0.1011(0.0312)$ & 0.2316 \\
$\rho_{v, 3}(-0.1)$ & $-0.0959(0.0261)$ & 0.1945 \\
$\rho_{v, 4}(-0.1)$ & $-0.1003(0.0292)$ & 0.1824 \\
$\rho_{1,2}(0.5)$ & $0.4960(0.0401)$ & 0.0079 \\
$\rho_{1,3}(0.5)$ & $0.4775(0.0405)$ & 0.0448 \\
$\rho_{1,4}(0.5)$ & $0.4951(0.0326)$ & 0.0098 \\
$\rho_{2,3}(0.5)$ & $0.4978(0.0393)$ & 0.0043 \\
$\rho_{2,4}(0.5)$ & $0.5012(0.0309)$ & 0.0024 \\
$\rho_{3,4}(0.5)$ & $0.4932(0.0409)$ & 0.0135 \\
\hline
\end{tabular}

constant over the time windows. For this reason Table 1 shows the average over $q$ of the estimated parameter values. To complete this analysis, the third column of Table 1 shows the average relative errors of the parameters which is computed as follows:

$$
E_{z}=\frac{1}{16} \sum_{q=1}^{16} \frac{\left|z_{q}-z^{*}\right|}{\left|z^{*}\right|}
$$

where $z^{*}$ is the true value of the investigated parameter and $z_{q}$ the estimated value of $z^{*}$ obtained solving the $q$-th estimation problem. We observe that all parameters are approximated with at least two correct significant digits except for the correlation parameters $\rho_{v, i}$ which are approximated with only one correct significant digit.

\section{The performance of the estimation procedure on empiri- cal data: an analysis on bond yields}

In this section we study the ability of the stochastic volatility model to describe bond yields. Specifically, we face two kinds of problems. One is calibrating the model on different yields having the same maturity (see application (a) in Section 2). Here the analysis focuses 
on the interactions among different countries belonging to the same geographical area with the aim of analyzing convergence/divergence of sovereign yields in Europe and developing an early warning indicator. The other is calibrating the model on German yields with different maturities (see application (b) in Section 2). This second analysis allows us to verify the robustness of the calibrated model in reproducing the stylized facts of the yield curves.

\subsection{The analysis of government bond yields in the Eurozone}

In this experiment we calibrate the stochastic volatility model (1)- (2) on four yields representing different European countries and on the EONIA interbank rate which can be considered as a proxy of the European monetary policy (see Giannone et al. 2011, Mesters et al. 2014). Here $x_{i, t}, i=1,2, \ldots, 4$, denote the yields of the four European countries and $x_{5, t}$ denotes the EONIA rate at the current time $t$.

We use the daily values of four bond yields with three month maturity: the German GETBT1 index (Germany 3 Month Bubill Maturin), the French GBTF3MO index (France Treasury Bills 3 Months), the Italian GBOTG3M index (Italy Bots Treasury Bill 3 Months) and the Spanish GSPG3M index (Spanish Govt Generic Bonds 3 Months) ${ }^{9}$. We have chosen these yields because they represent different geographical areas of the Eurozone. The data run from 29 March, 2004 to 3 April, 2014, corresponding to 2617 trading days.

Figure 3 shows the percentage of the yield time series (left panel) and of the EONIA rate (right panel) used in the calibration procedure. In simplified terms, the time series considered can be distinguished in several phases: first, a pre-crisis phase, stretching until the beginning of 2006, in which bond yields and EONIA are quite stationary and no large discrepancies among countries are observable; second, a phase of tension where the economic system comes under pressure from uncertain valuations of the sub-prime mortgages which lead to an increase of the indices and the interbank interest rate; third, a phase starting with the collapse of Lehman Brothers in September 2008, resulting in an abrupt collapse of all series; and, finally, a phase beginning with the outbreak of the sovereign debt crisis in May 2010 (see Eser et al. 2012 for further details). This phase puts some economic systems in the Eurozone area under enormous stress due to their exposure to sovereign debt. This last phase is reflected in the rise of Italian and Spanish yields and peaks at the end of 2011, when the yields of these two Mediterranean countries diverge from those of other European countries.

Figure 3 gives us further important information: the minimum value of the GETBT1

\footnotetext{
${ }^{9}$ The three month yield data are from Bloomberg and the EONIA data are freely downloadable from http://www.emmi-benchmarks.eu/euribor-eonia-org/eonia-rates.html.
} 
index (Germany), GBTF3MO index (France) and the EONIA rate are negative and equal to $-0.205,-0.105$ and -0.205 respectively. This implies that by calibrating $\tilde{m}$ in the stochastic volatility model (1)- (2), the parameter should return a value equal to zero, which allows for negative yields. In confirmation of this, the value of $\tilde{m}$ which maximizes the likelihood function is zero. Consequently, in this experiment, we calibrate the stochastic volatility model with $\tilde{m}=0$.
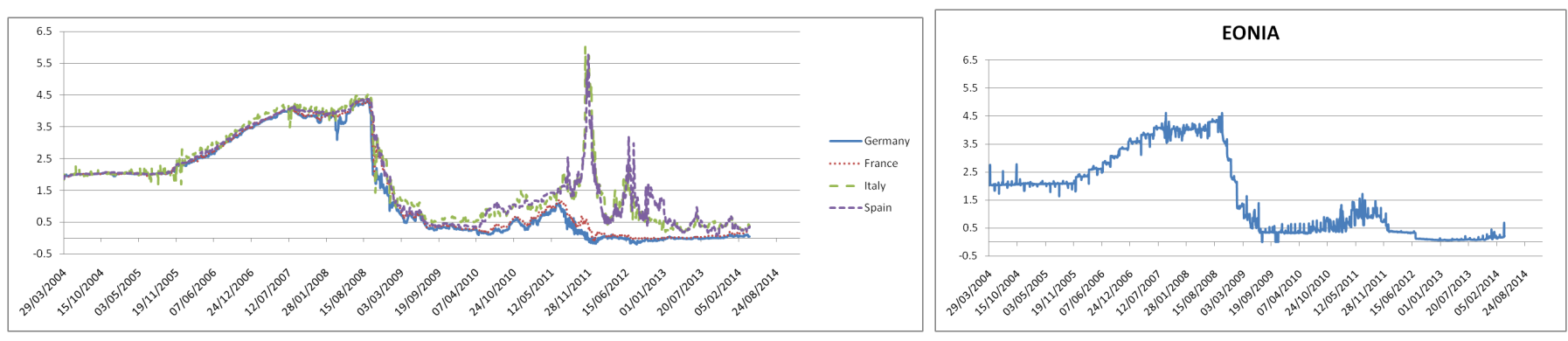

Figure 3: Three month yield GETBT1 index (Germany), GBTF3MO index (France), GBOTG3M index (Italy), GSPG3M index (Spain), left side; EONIA interbank rate, right side, as function of time.

\subsection{The estimation procedure}

In order to estimate the model parameters we use a time window with $m_{o b s}$ number of consecutive observations for each of the five series. Specifically, the model parameters are calibrated every month using a year of past observations (i.e. $m_{o b s}=260$ ). After each month, we solve the calibration problem again adding the 22 new daily observations and discarding the 22 oldest ones. In this manner the length of the time window used in the calibration is kept constant. Hence, we solve 105 calibration problems and the solution of these problems provide a monthly historical series of each model parameter.

Table 2 shows average values and standard deviations of the model parameters obtained by the estimation procedure. Specifically, the confidence interval of the estimated values of the parameters in Table 2 are obtained running 100 trajectories for each index. These trajectories are obtained perturbing each index by adding a noise sampled from a normal distribution with zero mean and standard deviation given by $\gamma_{n}=\xi \gamma_{s}$. The quantity $\gamma_{s}$ is the standard deviation of the observed data and $\xi$ is a constant also known as "noise to signal ratio" (see, for example, Nielsen and Frederiksen 2007, Recchioni et al. $2015)$. We set the noise to signal ratio, $\xi$, equal to $1 \%$. For each of the 100 trajectories we solve 105 calibration problems and the results are shown in the Table 2 . The first column of the Table lists the names of the parameters. Here, the indices G, F, I, S and E refer to Germany, France, Italy, Spain and EONIA respectively. The values shown in Table 2 
Table 2: Parameter name (first column); average values of the parameters (second column); average standard deviations of the estimated parameters (third column). All the average values are computed by using the 105 estimated values of the parameters coming for the solution of the estimation problems on the 100 trajectories.

\begin{tabular}{ccc}
\hline Parameter name & Ave. value & Ave. St. Dev. \\
\hline$\epsilon$ & 0.0142 & 0.00001 \\
$\theta$ & 0.014031 & 0.0000016 \\
$\chi$ & 0.04984 & 0.0000071 \\
$v_{0}$ & 0.49303 & 0.0062056 \\
$\sigma_{G}$ & 0.8524 & 0.003948 \\
$\sigma_{F}$ & 0.8169 & 0.004632 \\
$\sigma_{I}$ & 2.2088 & 0.001066 \\
$\sigma_{S}$ & 1.6490 & 0.006435 \\
$\sigma_{E}$ & 2.2181 & 0.00626 \\
$\rho_{v, G}$ & 0.03253 & 0.000031 \\
$\rho_{v, F}$ & 0.01977 & 0.000031 \\
$\rho_{v, I}$ & 0.04141 & 0.000015 \\
$\rho_{v, S}$ & 0.02711 & 0.000041 \\
$\rho_{v, E}$ & 0.09795 & 0.000016 \\
$\rho_{G, F}$ & 0.0992 & 0.001194 \\
$\rho_{G, I}$ & 0.0159 & 0.000777 \\
$\rho_{G, S}$ & 0.0775 & 0.001495 \\
$\rho_{G, E}$ & -0.0021037 & 0.0007807 \\
$\rho_{F, I}$ & 0.02820 & 0.0007873 \\
$\rho_{F, S}$ & 0.081493 & 0.001818 \\
$\rho_{F, E}$ & 0.00857 & 0.0008217 \\
$\rho_{I, S}$ & 0.07518 & 0.000969 \\
$\rho_{I, E}$ & 0.0008828 & 0.0004008 \\
$\rho_{S, E}$ & 0.032588 & 0.001141 \\
\hline & &
\end{tabular}

confirm that all parameters are statistically significant.

In order to assess the robustness of our estimation procedure, we evaluate the sensitivity of the estimated parameter values (see Table 2) first with respect to the number of observations, $m_{o b s}$, used in the time window and, second, to the EONIA effect.

Firstly, we estimate the model parameters in a time windows of $m_{\text {obs }}=130$ observations. We compare these values with those obtained with $m_{o b s}=260$ by applying the two-sample Kolmogorov-Smirnov (KS) goodness-of-fit hypothesis test. The test confirms that the historical series of model parameters, estimated using the two samples, are drawn from the same population at a significance level of 0.05 .

Secondly, we analyze the robustness of the parameters shown in Table 2 with respect to 
the EONIA. In this regard, we estimate the stochastic volatility model (1)- (2) without the EONIA. As in the previous case, we compare the time series of the new estimated values with those with the EONIA by implementing the KS test at a significance level of 0.05. Also in this case, the KS test never rejects the null hypothesis ${ }^{10}$. The fact that the estimated values of the model parameters do not vary when introducing EONIA indicates the model's ability to describe the market behavior through the parameter values. Given that the introduction of the EONIA does not generate different information from that obtained using only the four bond yields we can conclude that the stochastic volatility model already fully captures all the information contained in the data. The EONIA, in fact, commonly accepted as a measure of the ECB's non-standard monetary policy (see Borio and Disyata 2010), must somehow already be included in the yield time series and, therefore, should not generate significant changes in the parameter estimation.

We now analyze the model ability to fit the empirical data distributions. Thanks to Eqs. (99)-(101), we are able to compute the expected value, the variance, the skewness and the kurtosis (see Appendix $\mathrm{C}$ for the analytical derivation of moments). In Table 3 we compare the theoretical moments with the empirical ones arising from the yield and EONIA time series.

Table 3: Empirical and theoretical moments of monthly yields.

\begin{tabular}{|c|c|c|c|c|}
\hline empirical (theoretical) moments & Mean & Variance & Skewness & Kurtosis \\
\hline Germany $(\%)$ & $1.545(1.526)$ & $2.026(2.027)$ & $0.367(0.368)$ & $-1.411(-1.414)$ \\
\hline France (\%) & $1.633(1.615)$ & $1.984(1.967)$ & $0.369(0.372)$ & $-1.383(-1.382)$ \\
\hline Italy $(\%)$ & $2.018(1.970)$ & $1.480(1.470)$ & $0.376(0.420)$ & $-1.147(-1.139)$ \\
\hline Spain (\%) & $1.989(1.957)$ & $1.403(1.421)$ & $0.393(0.423)$ & $-1.112(-1.127)$ \\
\hline Eonia $(\%)$ & $1.727(1.684)$ & $1.922(1.899)$ & $0.376(0.383)$ & $-1.344(-1.361)$ \\
\hline
\end{tabular}

As the reader can see, the theoretical moments reproduce the empirical ones very well.

\subsection{Bi-directional relationship across European countries}

A relevant question, notably for policy makers, is what are the relations among European countries and how can these relations be expressed through the sovereign yield curves. Since the creation of the Euro, the literature has analyzed the reactions of the different countries to common economic policies or shocks that have hit the Euro area. Most of

\footnotetext{
${ }^{10}$ The KS test results of each experiment and their $p$ value are available under request.
} 
the analyses have focused on the convergence (divergence) of government bond yields in Europe, especially those of the Euro area countries (see e.g Attinasi et al. 2011; Haugh et al. 2009; Sgherri and Zoli, 2009; Manganelli and Wolswijk, 2009; Barrios et al. 2009, Rault and Afonso, 2011; Afonso and Strauch, 2007).

We contribute to this literature by analyzing the interlinkages among the Euro countries investigated, their reaction to a common monetary policy and to various shocks that have hit the European economies since 2004. In this regard we study the specific country volatility, $\sigma_{j}$, since, as suggested by the empirical and theoretical literature, the volatility describes the macroeconomic uncertainty very well. In fact, many economies that have experienced a dramatic increase in volatility have also showed a greater economic vulnerability and uncertainty (see Baum et al. 2004; Ghosal \& Loungani, 2000; Grilli et al. 2014a, 2014b).

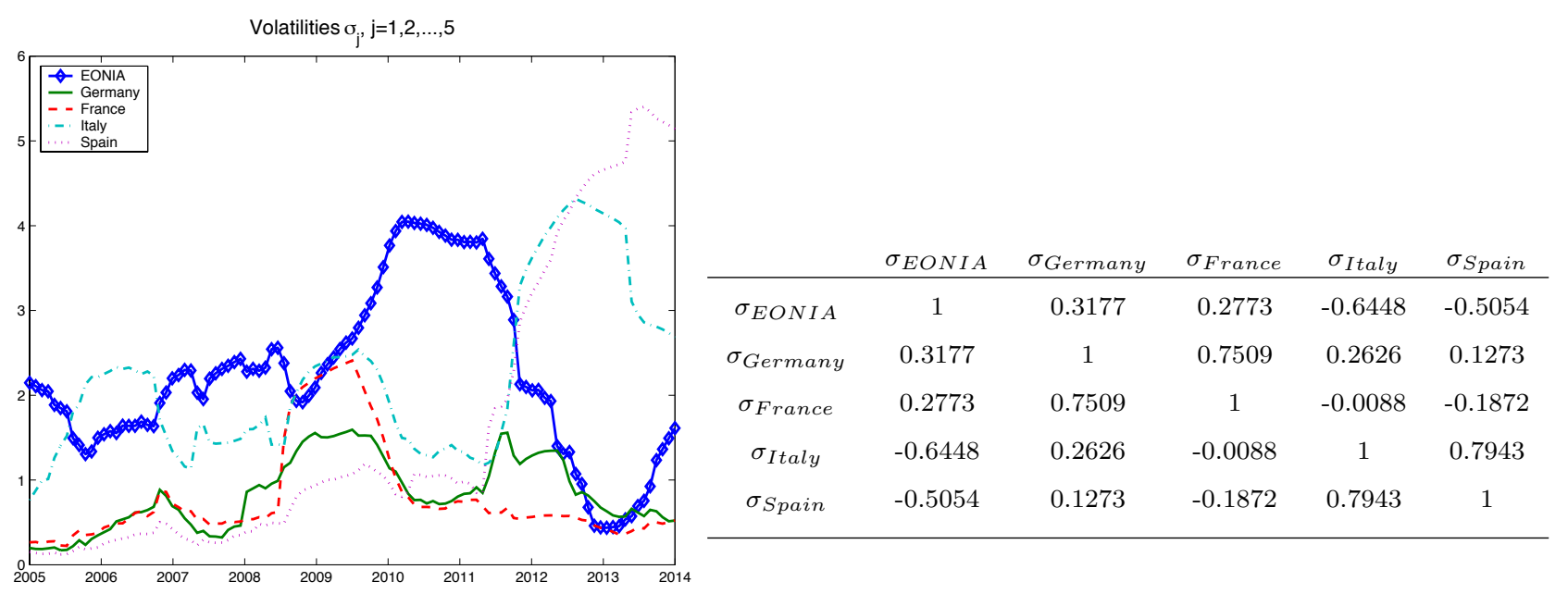

Figure 4: Time series of the average volatilities, $\sigma_{j}$, of EONIA (blue square line), Germany (green solid line), France (red dashed line), Italy (cyan dash-dotted line) and Spain (violet dotted line) over 100 trajectories (left side). Correlations among the specific volatilities at a $5 \%$ confidence level (right side). Colors are available on the web site version.

Figure 4 (left side) shows the time series of the specific country volatilities. As mentioned above, the parameter time series is obtained by solving 105 estimation problems for each of the 100 trajectories. Figure 4, therefore, shows the average value of the estimated $\sigma_{j}$ over 100 simulations. As the figure shows, the volatilities of Germany, France and Spain are very similar and remain low until 2008. By contrast, Italy's gross national debt increases between 2005 and 2006 (see Erber 2011) and this results in a volatility bubble in our model (see dash-dotted line in the left panel of Fig. 4). The turbulence in the financial markets is evident after the crisis of 2008. In fact, we can see a sharp rise in volatility first 
in Germany and, then, in the other countries. The bubble reaches its maximum at the end of 2009 when the European central bank intervenes by injecting liquidity and cutting the refinancing rates (see Eser et al. 2012). The action of the ECB corresponds, on the one hand, to a rapid decrease in bond yield volatilities of the Eurozone, and on the other hand, to a sharp rise in the EONIA volatility. This situation remains unchanged until the first quarter of 2011, when the volatility of Italy and Spain dramatically increases due to the sovereign debt crisis. Last but not least, it is worth noting that during the sovereign-debt crisis the French and German yield volatility returns to approximately the pre-crisis level. In contrast, the Spanish specific volatility parameter continues to increase in the first months of 2014, suggesting that this country is still under pressure. Similar results are also observed in the empirical study of Mesters et al. (2014).

The financial crisis is a clear case of materialisation and propagation of systemic risk. Furthermore, in the Euro area, the systemic risk has generated contagion which plays a crucial role in exacerbating the sovereign debt problems. Contagion is, in fact, a source of externality. The high volatility exhibited in the sovereign yield curves may be caused indirectly by spillovers originating in other sovereign countries.

In order to understand the hidden relationship among the sovereign country debts, we investigate the correlation among the specific volatility time series (see right panel of Fig. 4). We can observe a high correlation, equal to 0.75 , between the volatility of Germany and France, suggesting a strong two-way interaction between government finances. Moreover, correlations suggest that Germany holds significant amounts of Italian and Spanish sovereign bonds on its balance sheets. These exposures may easily lead to valuation losses and solvency concerns when sovereign yields rise sharply. On the other hand, France is negatively correlated with the volatilities of Italy and Spain indicating that this country is not affected by the risk in these two Mediterranean countries. Last but not least, the negative correlations between the EONIA volatility and the volatilities of the two Mediterranean countries indicate that the ECB monetary policies have lowered the riskiness of these two countries.

\subsection{An Early Warning indicator}

We now analyze the importance of the estimated volatility parameters, $v_{0}$, $\epsilon$, in capturing the instability in the government bond yields. Specifically, starting from the analysis of these parameters, we build an early warning indicator for crises. As already mentioned, the two parameters reflect the initial volatility and the volatility of volatility. By setting $\epsilon=0$ in Eq. (2), we obtain an ordinary differential equation whose solution is the variance of the yields given by: $v_{t}=v_{0} e^{-\chi\left(t-t_{0}\right)}+\theta\left(1-e^{-\chi\left(t-t_{0}\right)}\right)$, where $t_{0}$ is the initial time and 
$v_{0}$ is the initial volatility. In this circumstance, therefore, the volatility is deterministic (i.e. the market is in a "calm" situation) and the variance is known. Clearly, even when $\epsilon$ equals zero an increase of $v_{0}$ implies an increase of $v_{t}$ and this generates a positive/negative change in the yield movement. However, a positive value of $\epsilon$ indicates turbulence in the variance and this can be an indicator of the market instability.
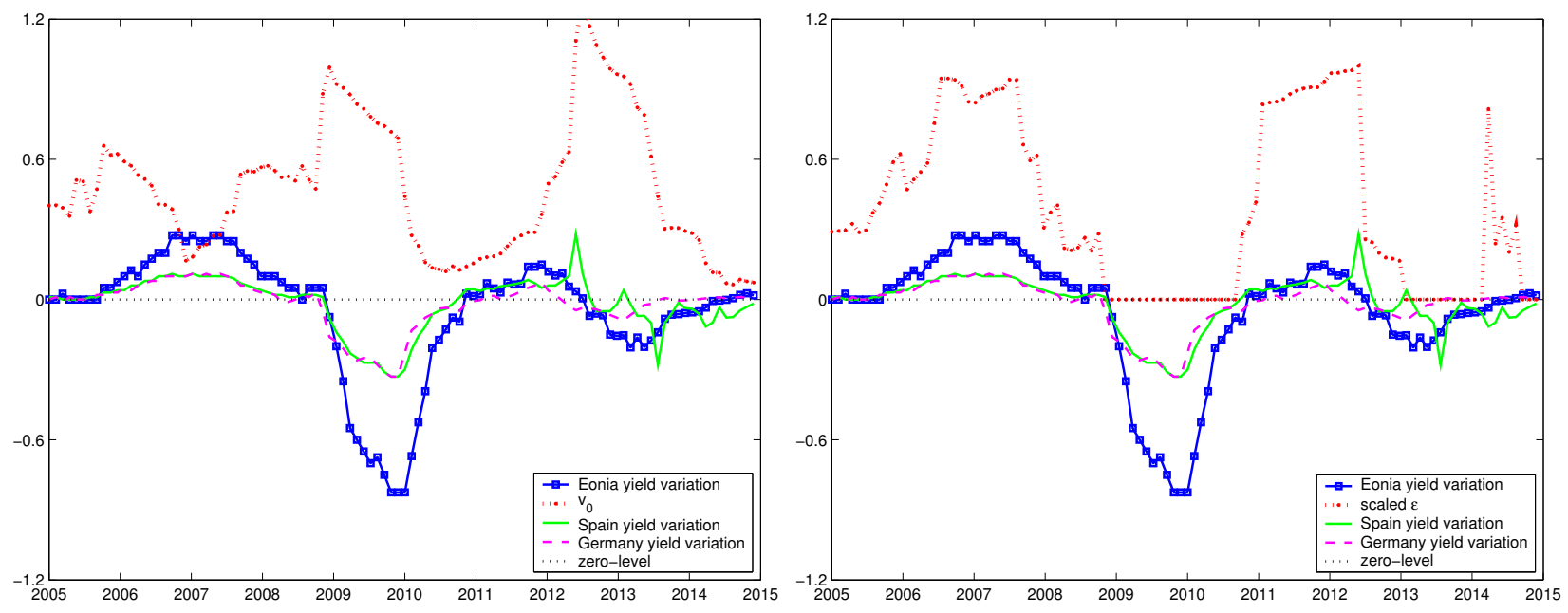

Figure 5: Time series of the average initial volatility, $v_{0}$, (left side) and average re-scaled vol of vol, $\epsilon$, (right side) over 100 trajectories (red dotted lines). Time series of yields variation of EONIA (blue square line), Spain (green solid line) and Germany (violet dashed line). Colors are available on the web site version.

Figure 5 shows the time series of the estimated volatility parameters, $v_{0}$ (left side) and the re-scaled ${ }^{11} \epsilon$ (right side), with the corresponding yield variations ${ }^{12}$. As the empirical analysis shows, $v_{0}$ (red dotted line in the left panel of Fig.5) is affected by the yield variations, with a good ability to predict their negative changes. This parameter, in fact, anticipates the two major yield downfalls that occur at the end of 2008 and 2011. Otherwise, $\epsilon$ (red dotted line in the right panel of Fig.5) increases only in the presence of positive yield variations, while it is zero for negative variations. In order to simultaneously capture the two effects, we consider the product $v_{0} \epsilon$. The left hand panel of Figure 6 shows the time series of $v_{0} \epsilon$ (red dotted line) and the yield variations.

The product of the two parameters, $\epsilon v_{0}$, identifies three bubbles: the first one peaks around June 2007, the second one peaks in the first months of 2008 and the last one increases in the beginning of 2011 and attains its maximum at the end of that year.

\footnotetext{
${ }^{11}$ The vol of vol $\epsilon$ has been re-scaled to make the figure more readable. Here, to understand the magnitude of the parameter, we report its summary statistics: mean 0.014, median 0.010, min 0, max 0.036 and st.dev 0.013 .

${ }^{12}$ We omit the yield variations of Italy and France, on the one hand, to make the figure more readable; on the other hand, because very similar to those of Spain and Germany, respectively.
} 


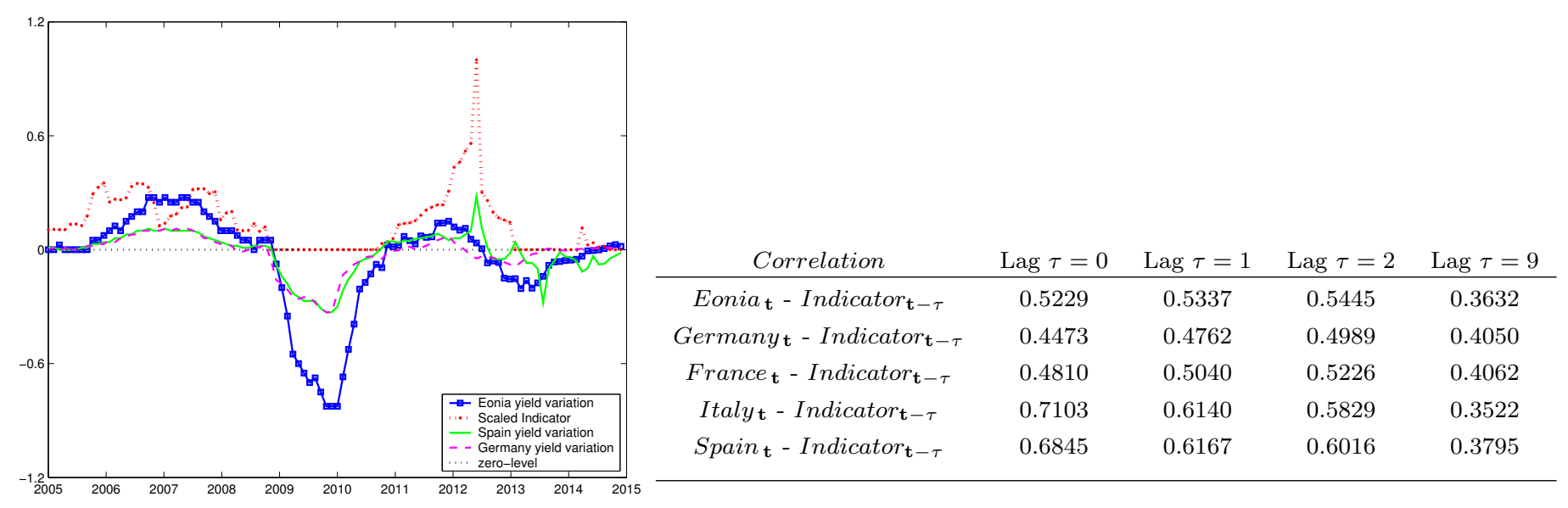

Figure 6: Left panel: Time series of the re-scaled product $v_{0} \epsilon$ (red dotted lines) and yields variation of EONIA (blue square line), Spain (green solid line) and Germany (violet dashed line). Right panel: correlations between the yield variations and the indicator $v_{0, t-\tau} \epsilon_{t-\tau}$, with $\tau=0,1,2,9$. Colors are available on the web site version.

Interestingly, the three bubbles seem to anticipate the three episodes of tension which characterize our time series. That is, the sub-prime mortgage, the collapse of Lehman Brothers and the sovereign debt crisis.

In order to verify whether this product can be considered an indicator capable of anticipating the instability of the Eurozone, we measure the correlations between the time series of the yield variations and the lagged time series of this product. The right hand side of Figure 6 shows these correlations. As the reader can notice, correlations increase at one-two lags (i.e. one-two months) before the abrupt change in the yields and they decrease at zero lag. In contrast, the correlations in the case of Italy and Spain remain high even at zero lag, demonstrating that these are areas of high instability.

In order to test the predictive power of the indicator, $v_{0} \epsilon$, we repeat the same exercise on the U.S market. The technique is as in the previous experiment, with the only exception that the data are now the U.S. treasury bills with different maturities. Therefore, the variables $x_{i, t}, i=1,2, \ldots, 5$ denote, respectively, the 3 -month $\left(x_{1, t}\right), 6$-month $\left(x_{2, t}\right), 1$ year $\left(x_{3, t}\right)$, 2-year $\left(x_{4, t}\right)$ and 5 -year $\left(x_{5, t}\right)$ yields at time $t$ as in application (b) in Section 2. The dataset considered ${ }^{13}$ covers the period from 29 March 2004 to 11 December 2014, which is slightly longer than the one used for the European indicator running from the 29

\footnotetext{
${ }^{13}$ Data are freely downloadable from the website: http://www.federalreserve.gov/releases/h15/data.htm. We consider treasury constant maturity series. These yields on actively traded non-inflation-indexed issues are adjusted to constant maturities. The historical adjustment factor can be found at www.treasury.gov/resource-center/data-chart-center/interest-rates/.
} 
March 2004 to 3 April 2014. Given the different structure of the U.S. data in terms both of business cycles and maturities, this experiment allows us to investigate the robustness of the early warning indicator in a different framework.

The left panel of Fig. 7 shows the time series used to estimate the model parameters. The middle panel shows the early warning indicator of the U.S. market as a function of time. As for the Eurozone, the indicator is given by the product between $v_{0}$ and $\epsilon$. We note that the U.S. indicator is able to anticipate the two main turbulences in the U.S financial market, namely the tension characterizing the sub-prime mortgage with a bubble in the middle of 2007 and a second peak in the first months of 2008 corresponding to the collapse of Lehman Brothers. By comparing the American and European ${ }^{14}$ indicators, shown in the right panel of Fig.7, we also notice how the U.S. crisis is more severe during these two episodes. During the two periods, in fact, the U.S indicator reaches peaks much higher than those of the European one. Moreover, as expected, the American indicator is not affected at all by the sovereign debt crisis which instead so much afflicts Europe. Last but not least, it is worth noting that the U.S. indicator increases again at the end of 2014 . This may indicate a new phase of instability in the U.S market. In this respect, nothing can be said on the European financial market given that the data only go up to the early months of 2014 .
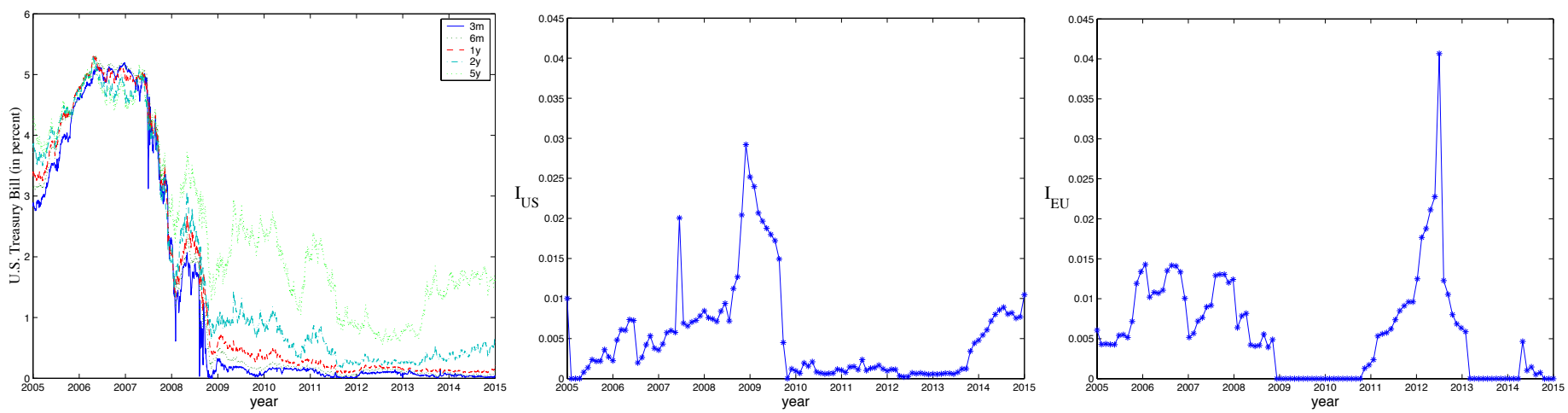

Figure 7: U.S. treasury bills (left panel); U.S. early warning indicator (middle panel) and E.U indicator (right panel) as function of time.

To conclude the section, we verify the capacity of the U.S. warning indicator to anticipate the instability of the American market. To this end, Table 4 shows the correlations between the time series of the yield variations for each maturity and the lagged time se-

\footnotetext{
${ }^{14}$ The E.U. indicator in the right panel of Fig. 7 corresponds to Fig. 6 but without rescaling the volatility parameters.
} 
ries of the indicator. As is clear, the correlation is positive for each yield but decreases with increasing maturity. Moreover, as the correlations indicate, only the three-month yields have anticipatory power. In fact, their correlations increase at one lag (i.e. one month) before the abrupt change in the yields, and they decrease at zero lag. For yields at higher maturity, however, the correlation decreases with lag, thus implying that the indicator signals the crisis when it is already in place. This result is open to two possible interpretations. On the one hand, short-maturity yields are considered more risky and therefore more related to market sentiment. On the other hand, investors perceive the two periods of economic turmoil as short-run events which do not affect the American long-run fundamentals.

Table 4: Correlations between the U.S. treasury bills variations and the indicator.

\begin{tabular}{ccccc}
\hline Correlation & Lag $\tau=0$ & Lag $\tau=1$ & Lag $\tau=2$ & Lag $\tau=9$ \\
$3-$ mont $_{\mathbf{t}}-$ Indicator $_{\mathbf{t}-\tau}$ & 0.5599 & 0.5691 & 0.5642 & 0.4544 \\
$6-$ mont $_{\mathbf{t}}-$ Indicator $_{\mathbf{t}-\tau}$ & 0.5549 & 0.5523 & 0.5429 & 0.4382 \\
$1-$ year $_{\mathbf{t}}-$ Indicator $_{\mathbf{t}-\tau}$ & 0.5431 & 0.5343 & 0.5215 & 0.4331 \\
$2-$ year $_{\mathbf{t}}-$ Indicator $_{\mathbf{t}-\tau}$ & 0.5060 & 0.4993 & 0.4920 & 0.4361 \\
$5-$ year $_{\mathbf{t}}-$ Indicator $_{\mathbf{t}-\tau}$ & 0.3226 & 0.3127 & 0.3117 & 0.1475 \\
\hline
\end{tabular}

\subsection{The analysis of German government bond yields with different ma- turities}

In this subsection we illustrate some preliminary results regarding the capacity of our stochastic volatility model to reproduce the bond yield term structure. Specifically, we show the model's robustness in estimating and forecasting bond yields with different maturities. To this end we calibrate our stochastic volatility model to German bond yields with three month, two and five year maturities. The estimation procedure is analogous to the one illustrated in Section 4.2 but with two important differences: (i) the variables $x_{j, t}$, with $j=1,2,3$, now denote the 3 -month, 2 -year and 5 -year German yields at time $t$ as in application (b) in Section 2 and (ii) the drift terms, $\mu_{i}$, are no longer set to zero but are integrable functions of time. Specifically, following Diebold and Li 2006, we choose the drift terms as follows:

$$
\int_{0}^{s} \mu_{j}\left(s^{\prime}\right) d s^{\prime}=\beta_{1, s}+\beta_{2, s}\left(\frac{1-e^{-\lambda_{s} \tau_{j}}}{\lambda_{s} \tau_{j}}\right)+\beta_{3, t}\left(\frac{1-e^{-\lambda_{s} \tau_{j}}}{\lambda_{s} \tau_{j}}-e^{-\lambda_{s} \tau_{j}}\right), j=1,2,3,
$$

where $\tau_{1}=3 / 12, \tau_{2}=2, \tau_{3}=5$ are the yield maturities expressed in years, parameters $\beta_{1, s}, \beta_{2, s}$ and $\beta_{3, s}$ represent, respectively, a long-term, a short term and a medium term 
factor, and $\lambda_{s}$ governs the exponential decay rate.

As in the previous experiment, we calibrate the model parameters each month using a year of past observations (i.e $m_{o b s}=260$ ). Moreover, the dataset period is from 29 March 2004 to 3 April 2014 (i.e 2617 trading days) as in Subsection 4.1. In contrast to the previous experiment, the value of $\tilde{m}$, which now maximizes the likelihood function, is equal to 1 . Consequently, in this experiment, we calibrate the stochastic volatility model with $\tilde{m}=1$. This difference is motivated on the one hand by the different dataset now incorporating long maturity yields which are not negative and, on the other hand, by the different model now including the drift terms.

Table 5 shows average values and standard deviations of the model parameters obtained by the estimation procedure on German government bond yields. The three month, two and five year maturities are denoted by $3 \mathrm{~m}, 2 \mathrm{y}$ and $5 \mathrm{y}$, respectively. As in Table 2, the average standard deviations of the estimated parameters are obtained running 100 trajectories for each yield. The low values of the standard deviations confirm the robustness

Table 5: Parameter name (first column); average values of the parameters (second column); average standard deviations of the estimated parameters (third column). All the average values are computed by using the 105 estimated values of the parameters coming for the solution of the estimation problems on the 100 trajectories.

\begin{tabular}{ccc}
\hline Parameter name & Ave. value & Ave. St. Dev. \\
\hline$\epsilon$ & 0.0125 & 0.00053 \\
$\theta$ & 0.0149 & 0.0000095 \\
$\chi$ & 0.0495 & 0.000012 \\
$v_{0}$ & 1.2859 & 0.009460 \\
$\sigma_{3 m}$ & 1.0296 & 0.004756 \\
$\sigma_{2 y}$ & 0.9619 & 0.0023342 \\
$\sigma_{5 y}$ & 0.9798 & 0.0013048 \\
$\rho_{v, 3 m}$ & 0.0217 & 0.00039 \\
$\rho_{v, 2 y}$ & 0.0232 & 0.00075 \\
$\rho_{v, 5 y}$ & 0.0219 & 0.00025 \\
$\rho_{3 m, 2 y}$ & 0.1741 & 0.00433 \\
$\rho_{3 m, 5 y}$ & 0.1712 & 0.00621 \\
$\rho_{2 y, 5 y}$ & 0.2548 & 0.00951 \\
$\beta_{1}$ & 0.0119 & 0.00134 \\
$\beta_{2}$ & 0.0264 & 0.00323 \\
$\beta_{3}$ & -0.0482 & 0.000191 \\
$\lambda$ & 0.00007 & 0.00003 \\
\hline
\end{tabular}

of the model parameter estimation. Having successfully proven that our model correctly interprets the bond yield term structure, we now test its ability to forecast. In this regard, we present the one-month-ahead forecast values on the German government bond yields. 

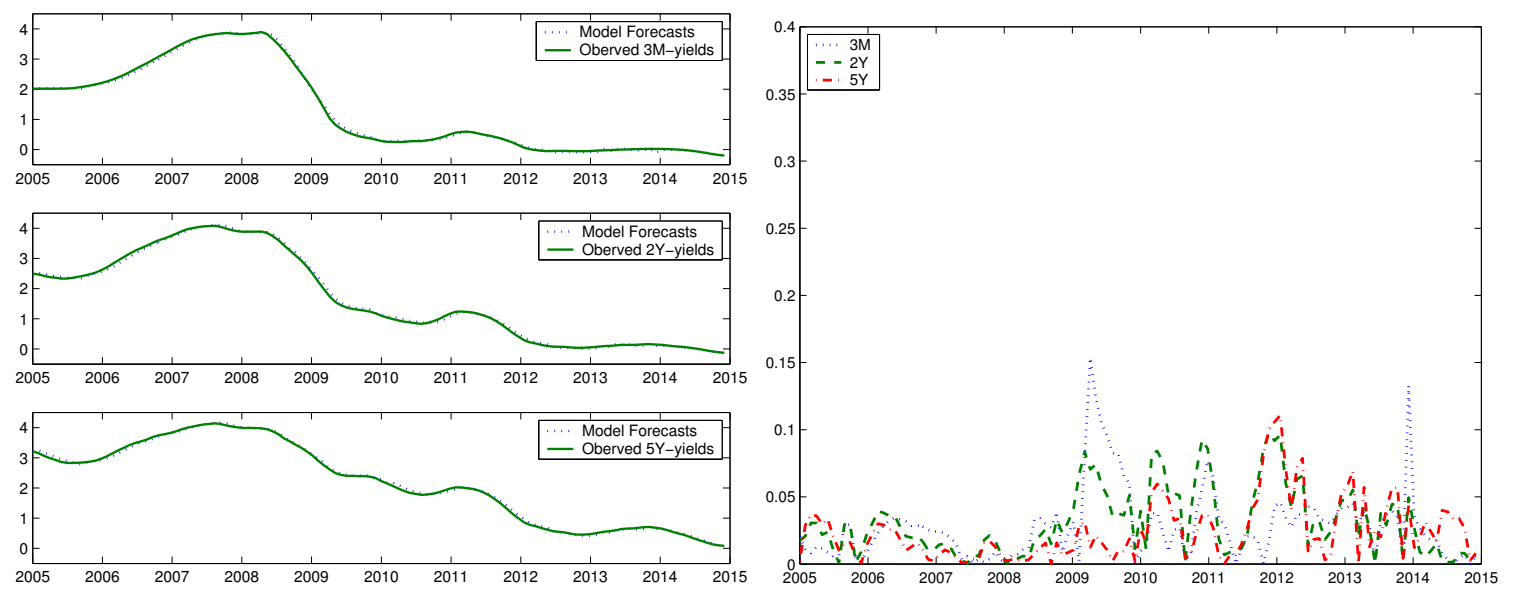

Figure 8: Left Panels: Observed and one month ahead forecast German yields with three month (top row), two and five years (central and bottom rows). Right Panel: Relative errors of the one month ahead bond yield forecasts shown in the left panels. Relative errors of three month yield forecasts in blue dotted line, of two year yield forecasts in green dashed line and five year yield forecast in dashed-dotted line. Colors are available on the web site version.

Specifically, we use the estimated model parameters at time $t$ to forecast the yields at time $t+\Delta t$, with $\Delta t=22 / 260$. The forecast of yields, $x_{j, t+\Delta t}$, is computed using the following equation:

$$
\begin{aligned}
& \hat{x}_{j, t+\Delta t}=x_{j, t}+\beta_{1, t}+\beta_{2, t}\left(\frac{1-e^{-\lambda_{t} \tau_{j}}}{\lambda_{t} \tau_{j}}\right) \beta_{2, t}+\beta_{3, t}\left(\frac{1-e^{-\lambda_{t} \tau_{j}}}{\lambda_{t} \tau_{j}}-e^{-\lambda_{t} \tau_{j}}\right) \\
& -\frac{1}{2} \sigma_{j}^{2}\left(\theta \Delta t+\left(v_{0}-\theta\right) \frac{\left(1-e^{-\chi \Delta t}\right)}{\chi}\right), j=1,2,3 .
\end{aligned}
$$

It is worth noting that when $\tilde{m} \neq 0$ and $\mu_{j} \neq 0$, Eq. 36 represents the conditional expected value of the stochastic model as shown in Eq. 102. The three panels on the left in Figure 8 show the forecast and true values of the yields for each maturity (i.e three month (top), two and five years (middle and bottom)). As shown in Figure 8, a forecasting process obtained by the conditional expected value of the stochastic model fits the observed values for all yields quite well. The accuracy of our forecasts is displayed in the right panel of Fig. 8, where we show the relative errors of one-month-ahead yield forecasts. Specifically, the panel shows the quantities $e_{r, t}=\left|\hat{x}_{j, t+\Delta t}-x_{j, t+\Delta t}\right| /\left|x_{j, t+\Delta t}\right|$ versus $t, t=0,1, \ldots, \tau$. Note that the larger relative errors are strongly affected by the turbulence in the yield time series. Specifically, the magnitude of the relative errors significantly increase at the end of 2008 with the collapse of Lehman Brothers. A similar situation occurs at the end of 2011, with the beginning of the sovereign debt crisis. However, we stress that the average relative error is 0.0276 . This means that, on average, the forecast values have 


\begin{tabular}{l|l|cc|c}
\hline \multicolumn{5}{c}{ Results using the forecast in Eq. (36). } \\
\hline yield maturity & $R^{2}$ & $\phi_{0}$ & $\phi_{1}$ & $P$ \\
\hline 3 months & 0.9962 & -0.1112 & 1.0296 & 0.9950 \\
2 years & 0.9973 & -0.2235 & 1.0520 & 0.9918 \\
5 years & 0.9966 & -0.1257 & 1.0283 & 0.9944 \\
\hline
\end{tabular}

Results using the naive forecast in Eq. (37).

\begin{tabular}{l|l|cc|c}
\hline yield maturity & $R^{2}$ & $\phi_{0}$ & $\phi_{1}$ & $P$ \\
\hline 3 months & 0.9886 & -0.4223 & 1.1060 & 0.9608 \\
2 years & 0.9923 & -0.4339 & 1.1036 & 0.9432 \\
5 years & 0.9907 & -0.4075 & 1.0978 & 0.8697 \\
\hline
\end{tabular}

Table 6: Results from regression (38) and the measure $P$ using the forecast in Eq. (36) (top panel) and in Eq. (37) (bottom panel).

two significant correct digits (i.e the average relative error is smaller than $5 \cdot 10^{-2}$ ). The relative errors shown in Figure 8 indicate that the forecast values are able to match the observed prices even when they are affected by abrupt changes. Moreover, the results of one-month-ahead forecast values obtained using formula (36) are compared with those of a naive forecast. Specifically, the naive forecast, which just states that tomorrow will be like today, can be written as:

$$
\hat{x}_{j, t+\Delta t}^{n}=x_{j, t}, j=1,2,3 .
$$

We compare the informational content of forecasts obtained through the two mentioned equations via the regression-based method test (see Poon and Granger 2003). We regress the "actual" observed $x_{j, t+\Delta t}^{o}$, on the forecast, $\hat{x}_{j, t+\Delta t}^{f}$ as follows:

$$
x_{j, t+\Delta t}^{o}=\phi_{0}+\phi_{1} \hat{x}_{j, t+\Delta t}^{f}+\xi_{j, t+\Delta t}, j=1,2,3,
$$

where $\hat{x}_{j, t+\Delta t}^{f}$ is or the forecast obtained via Eq. (36), or the naive forecast in Eq. (37). As stressed in Poon and Granger 2003, three conditions are required to obtain a good prediction. Firstly, the forecast is reliable when the $R^{2}$ in the regression approaches one. Secondly, the prediction is unbiased if $\phi_{0}$ and $\phi_{1}$ approach zero and one, respectively. Thirdly, the explanatory power of the forecasts ${ }^{15}$ is high when $P=1-\frac{\sum_{t}\left(x_{j, t+\Delta t}^{o}-x_{j, t+\Delta t}^{f}\right)^{2}}{\sum_{t}\left(x_{j, t+\Delta t}^{o}-\mu_{x}\right)^{2}}$ is close to one ${ }^{16}$.

Table 6 shows that the forecasting process obtained using the conditional expected value satisfies all three conditions of reliability and always outperforms the naive forecast.

\footnotetext{
${ }^{15}$ The explanatory power of forecasts is defined as a comparison between the amount of variation in the forecast errors and the variation in the observed yields.

${ }^{16} \mu_{x}$ in $\mathrm{P}$ is the mean value of the observed yields.
} 
As previously mentioned, this is only a preliminary analysis on the use of this model to study the term structure of yields. However, the results seem to be encouraging.

\section{Concluding remarks}

In this paper we have presented a hybrid Heston model with local volatility. The model is analytically tractable, allowing us to derive the marginal conditional probability density function and explicit elementary formulas for the moments of the yield variables. Furthermore, in order to to cope with the curse of dimensionality that arises when an efficient calibration of the model is necessary, an expansion of the marginal conditional probability density function is proposed. This perturbation approach can be applied to several stochastic models and allows reliable time series of the model parameters to be obtained, which in turn provide us with two contributions.

We conducted an empirical analysis on two different datasets in order to asses the ability of the stochastic volatility model to describe and forecast bond yields. The first dataset consists of the three month government bond yields in the Eurozone (i.e. Germany, France, Italy and Spain) plus the EONIA interbank rate; the second one consists of German government bond yields with different maturities (i.e. three months, two and five years). The empirical analysis on the first dataset shows that the model captures changes in the yield volatility and predicts future bond values very well.

This is mainly due to the simplicity of the model which, being analytically tractable, has allowed us to efficiently estimate the parameters via the maximum likelihood approach. The ability of the estimation procedure to capture the parameters and their changes over the time period considered has also been assessed on simulated time series mimicking the real data volatility, which confirmed the reliability of the results obtained.

Furthermore, we tested the ability of our procedure to reproduce some well-known phenomena of convergence/divergence among European countries. Our results confirm some empirical evidence already illustrated in other studies (see Mesters et al. 2014).

Last but not least, we analyzed the capacity of the estimated volatility parameters to capture and anticipate the instability of the government bond yields. To this end, we

have developed an early warning indicator, which seems to be able to anticipate phases of instability characterizing our time series. In order to better assess the performance of this indicator, it was tested on U.S. treasury bills with various maturities (3-month, 6-month, 1-year, 2-year and 5-year). The results of the empirical analysis on the U.S. data confirm the ability of the indicator in anticipating periods of strong financial instability.

The analysis on the second dataset (i.e. German bond yields with different maturities) is only preliminary. However, these preliminary results confirm the validity of the hybrid Heston model in interpreting bond yield term structure. In fact, also in this experiment, 
the model is able to describe and forecast bond yields. This preliminary analysis will be the object of future research.

\section{Acknowledgements}

The research leading to these results has received funding from the European Union, Seventh Framework Programme FP7, under grant agreement FinMaP no. 612955.

\section{Appendix A: An integral formula for the marginal conditional density $M$}

We derive the integral representation formula for the marginal probability density $M$ in Eq. (12). Following Duffie, Pan and Singleton 2000, the Fourier transform, $\hat{M}$, of the marginal conditional density $M$ in Eq. (11), is given by:

$$
\begin{aligned}
\hat{M}(s, \underline{k}, v)=\int_{\mathbb{R}^{n}} e^{\imath \underline{k}^{T}\left(\underline{x}-\underline{x}^{\prime}\right)} M\left(\underline{x}, v, t, \underline{x}^{\prime}, t^{\prime}\right) d \underline{x}^{\prime}= \\
\quad t<t^{\prime}, t, t^{\prime}>0, s=t^{\prime}-t>0, \underline{k} \in \mathbb{R}^{n}, v>0,
\end{aligned}
$$

and is the solution of the following problem:

$$
\frac{\partial \hat{M}}{\partial s}=\epsilon^{2} \frac{v}{2} \frac{\partial^{2} \hat{M}}{\partial v^{2}}-\imath \epsilon v b(\underline{k}) \frac{\partial \hat{M}}{\partial v}+\chi(\theta-v) \frac{\partial \hat{M}}{\partial v}-\left[\frac{v}{2}(a(\underline{k})-\imath c(\underline{k}))+\imath \underline{k}^{T} \underline{\mu}\right] \hat{M},
$$

with the initial condition:

$$
\hat{M}(0, \underline{k}, v)=1 .
$$

As suggested in Duffie, Pan and Singleton 2000, we seek $\hat{M}$ in the following form:

$$
\hat{M}(s, \underline{k}, v)=e^{A(s, \underline{k})-v B(s, \underline{k})}, s>0, \underline{k} \in \mathbb{R}^{n},
$$

where $A$ and $B$ are functions satisfying the following conditions:

$$
A(0, \underline{k})=B(0, \underline{k})=0, \quad \underline{k} \in \mathbb{R}^{n} .
$$

In order to determine $A, B$, we substitute Eq. (42) into Eq. (40). As a consequence, $A$ and $B$ satisfy the following ordinary differential equations:

$$
\begin{gathered}
\frac{d}{d s} A(s, \underline{k})=-\chi \theta B(s, \underline{k})-\imath \underline{k}^{T} \underline{\mu}, \\
\frac{d}{d s} B(s, \underline{k})=-\frac{\epsilon^{2}}{2} B^{2}(s, \underline{k})-(\chi+\imath \epsilon b(\underline{k})) B(s, \underline{k})+\frac{1}{2}(a(\underline{k})-\imath c(\underline{k})),
\end{gathered}
$$


with initial conditions:

$$
\begin{aligned}
& A(0, \underline{k})=0, \quad \underline{k} \in \mathbb{R}^{n}, \\
& B(0, \underline{k})=0, \quad \underline{k} \in \mathbb{R}^{n} .
\end{aligned}
$$

Now we solve the ordinary differential equations (44), (45) using a standard technique. We look for the solution of Eq. (45) in the following form:

$$
B(s, \underline{k})=\frac{\frac{d C}{d s}(s, \underline{k})}{C(s, \underline{k})}, s>0, \underline{k} \in \mathbb{R}^{n} .
$$

By substituting Eq. (48) into Eqs. (45) and (47), we obtain $C$ as the solution of the following problem:

$$
\frac{d^{2}}{d s^{2}} C+(\chi+\imath \epsilon b(\underline{k})) \frac{d}{d s} C-\frac{\epsilon^{2}}{4}(a(\underline{k})-\imath c(\underline{k})) C=0,
$$

with initial conditions:

$$
C(0, \underline{k})=1, \quad \frac{d C}{d s}(0, \underline{k})=0, \underline{k} \in \mathbb{R}^{n} .
$$

By solving problem (49), (50) we obtain:

$$
C(s, \underline{k})=\frac{(\zeta+\nu)}{2 \zeta} e^{(\nu-\zeta) s}+\frac{(\zeta-\nu)}{2 \zeta} e^{(\nu+\zeta) s}, s>0, \underline{k} \in \mathbb{R}^{n},
$$

and

$$
B(s, \underline{k})=\frac{2}{\epsilon^{2}} \frac{\left(\zeta^{2}-\nu^{2}\right)\left(1-e^{-2 s \zeta}\right)}{(\nu+\zeta) e^{2 s \zeta}+(\zeta-\nu)},
$$

where $\nu$ and $\zeta$ are in Eqs. (17)-(18). By using Eq. (48) into Eq. (44) we obtain:

$$
\frac{d}{d s} A(s, \underline{k})=-\chi \theta \frac{d}{d s} \ln \left(\frac{C(s, \underline{k})}{C(0, \underline{k})}\right)
$$

so that, by integrating Eq. (53) with respect to $s$, we obtain:

$$
A(s, \underline{k})=-\frac{2 \chi \theta}{\epsilon^{2}} s(\zeta+\nu)-\frac{2 \chi \theta}{\epsilon^{2}} \ln \left(\frac{(\zeta+\nu)}{2 \zeta} e^{-2 \zeta s}+\frac{(\zeta-\nu)}{2 \zeta}\right) .
$$

Substituting formulas (52) and (54) into Eq. (42) we obtain:

$$
\begin{aligned}
& \hat{M}(s, \underline{k}, v)= \\
& e^{-\imath s \underline{k}^{T} \underline{\mu}} e^{-\frac{v}{2}(a(\underline{k})-\imath c(\underline{k})) \varphi(s, \underline{k})} e^{-\frac{2 \chi \theta s}{\epsilon^{2}}(\nu(\underline{k})+\zeta(\underline{k}))} e^{-\frac{2 \chi \theta}{\epsilon^{2}} \ln \left(1+\frac{(\nu(k)+\zeta(k))}{2 \zeta(\underline{k})}\left(e^{-2 \zeta(\underline{k})}-1\right)\right)}, \\
& s>0, \underline{k} \in \mathbb{R}^{n}, v>0,
\end{aligned}
$$

where $\varphi$ is defined in Eq. (16). Finally, the marginal conditional probability density $M$ (see Eq. (12)) follows from Eq. (55) inverting the Fourier transform. 


\section{Appendix B: The series expansion in powers of the vol of vol}

We assume that the expansion (21) of $M$ in powers of $\epsilon$ holds. This implies that the following expansion in powers of $\epsilon$ holds for the Fourier transform of $M$ :

$$
\hat{M}(s, \underline{k}, v)=\sum_{j=1}^{+\infty} \epsilon^{j} \hat{M}_{j}(s, \underline{k}, v),
$$

where

$$
M_{j}\left(s, \underline{x}, \underline{x}^{\prime}, v\right)=\frac{1}{(2 \pi)^{n}} \int_{\mathbb{R}^{n}} e^{-\imath \underline{k}\left(\underline{x}-\underline{x}^{\prime}\right)} \hat{M}_{j}(s, \underline{k}, v) d \underline{k} .
$$

We derive the first wo terms of the series expansion (57) by computing the Taylor expansion with base point $\epsilon=0$ of the exponent of Eq. (55). Using some elementary computations, we obtain the following expansions as $\epsilon \rightarrow 0^{+}$(see Eqs. (18), (17)):

$$
\begin{gathered}
\zeta(\underline{k})=\frac{\chi}{2}+\frac{\imath b(\underline{k})}{2} \epsilon+\frac{\epsilon^{2}}{4}(a(\underline{k})-\imath c(\underline{k}))+\frac{\epsilon^{3}}{6}\left(-\frac{3 \imath b(\underline{k})}{2 \chi^{2}}\right)(a(\underline{k})-\imath c(\underline{k}))+o\left(\epsilon^{3}\right), \epsilon \rightarrow(\text { 68) }) \\
\zeta(\underline{k})+\nu(\underline{k})=\frac{\epsilon^{2}}{4 \chi}(a(\underline{k})-\imath c(\underline{k}))-\frac{\epsilon^{3}}{6}\left(\frac{3 \imath b(\underline{k})}{2 \chi^{2}}\right)(a(\underline{k})-\imath c(\underline{k}))+o\left(\epsilon^{3}\right), \epsilon \rightarrow 0^{+},(59) \\
\zeta(\underline{k})-\nu(\underline{k})=\chi+\imath b(\underline{k}) \epsilon+\frac{\epsilon^{2}}{4}(a(\underline{k})-\imath c(\underline{k}))+ \\
\frac{\epsilon^{3}}{6}\left(-\frac{3 \imath b(\underline{k})}{2 \chi^{2}}\right)(a(\underline{k})-\imath c(\underline{k}))+o\left(\epsilon^{3}\right), \\
\epsilon \rightarrow 0^{+},
\end{gathered}
$$

where $o(\cdot)$ is the Landau symbol. Using Eqs. (58)-(60), we obtain the following formulas:

$$
\begin{gathered}
\ln \left(1-\frac{(\zeta(\underline{k})+\nu(\underline{k}))\left(1-e^{-2 s \zeta(\underline{k})}\right)}{2 \zeta(\underline{k})}\right)=-\frac{\epsilon^{2}}{4}(a(\underline{k})-\imath c(\underline{k})) \frac{\left(1-e^{-\chi s}\right)}{\chi^{2}}+ \\
\frac{\epsilon^{3}}{6}\left(\frac{3 \imath b(\underline{k})}{\chi^{2}}\right)(a(\underline{k})-\imath c(\underline{k}))\left(\frac{1-e^{-\chi s}}{\chi}-\frac{s}{2}\right)+o\left(\epsilon^{3}\right), \epsilon \rightarrow 0^{+}, \\
\varphi(s, \underline{k})=\frac{1-e^{-2 s \zeta(\underline{k})}}{(\zeta(\underline{k})+\nu(\underline{k})) e^{-2 \zeta(\underline{k}) s}+(\zeta(\underline{k})-\nu(\underline{k}))}=\frac{1-e^{-\chi s}}{\chi}+\epsilon \frac{\imath b(\underline{k})}{\chi}\left(s-\frac{\left(1-e^{-\chi s}\right)}{\chi}\right)+ \\
\frac{\epsilon^{2}}{2}\left[\frac{(a(\underline{k})-\imath c(\underline{k}))}{\chi^{2}}\left(s-\frac{1-e^{-2 \chi s}}{2 \chi}\right)+\frac{2 b(\underline{k})^{2}}{\chi^{2}}\left(s-\frac{1-e^{-\chi s}}{\chi}\right)\right]+o\left(\epsilon^{2}\right),
\end{gathered}
$$


By substituting Eqs. (58), (59), (61) and (62) into Eq. (55), we obtain:

$$
\begin{gathered}
\hat{M}(s, \underline{k}, v)=e^{-\imath s \underline{k}^{T}} \underline{\mu} e^{-\frac{1}{2}(a(\underline{k})-\imath c(\underline{k})) f_{1}(s, v)} e^{\frac{\epsilon}{2} \imath b(\underline{k})(a(\underline{k})-\imath c(\underline{k})) f_{2}(s, v)+o(\epsilon),} \\
s>0, \underline{k} \in \mathbb{R}^{n}, v>0, \epsilon \rightarrow 0^{+}
\end{gathered}
$$

where $f_{1}$ are $f_{2}$ are the functions given in Eqs. (24)-(25). ¿From Eq. (63), we obtain the first two terms of the expansion in powers of $\epsilon$ of $\hat{M}$ :

$$
\begin{gathered}
\hat{M}_{0}(s, \underline{k}, v)=e^{-\imath s \underline{k}^{T} \underline{\mu}} e^{-\frac{1}{2}(a(\underline{k})-\imath c(\underline{k})) f_{1}(s, v)}, s>0, \underline{k} \in \mathbb{R}^{n}, v>0, \\
\hat{M}_{1}(s, \underline{k}, v)=e^{-\imath s \underline{k}^{T} \underline{\mu}} e^{-\frac{1}{2}(a(\underline{k})-\imath c(\underline{k})) f_{1}(s, v)}\left(\frac{\imath b(\underline{k})}{2 \chi}(a(\underline{k})-\imath c(\underline{k})) f_{2}(s, v)\right), \\
s>0, \underline{k} \in \mathbb{R}^{n}, v>0 .
\end{gathered}
$$

Now, by computing the inverse Fourier transform of $\hat{M}_{0}$ and $\hat{M}_{1}$ we come back to the variables $\underline{x}, \underline{x}^{\prime}$. In fact, from the definition of $a(\underline{k}), c(\underline{k}), \underline{k} \in \mathbb{R}^{n}$ (see Eqs. (13) and (15)) we obtain $\hat{M}_{0}$ as the Fourier transform of a multidimensional Gaussian function, given by:

$$
M_{0}\left(s, \underline{x}, \underline{x}^{\prime}, v\right)=\frac{e^{-\frac{1}{2 f_{1}(s, v)}\left(\underline{x}-\underline{x}^{\prime}+s \underline{\mu}-\frac{\tilde{m}}{2} f_{1}(s, v) \underline{\sigma}\right)^{T} \Gamma^{-1}\left(\underline{x}-\underline{x}^{\prime}+s \underline{\mu}-\frac{\tilde{m}}{2} f_{1}(s, v) \underline{\sigma}\right)}}{\sqrt{(2 \pi)^{n}\left(f_{1}(s, v)\right)^{n} \operatorname{det} \Gamma}} .
$$

In order to derive an explicit formula for $M_{1}$ we rewrite Eq. (65) as follows:

$$
\begin{aligned}
& M_{1}\left(s, \underline{x}, \underline{x}^{\prime}, v\right)= \\
& f_{2}(s, v) \frac{\imath}{2 \chi} \sum_{j=1}^{n} \sigma_{j} \rho_{v, j} \int_{\mathbb{R}^{n}} k_{j} e^{-\imath \underline{k}^{T}\left(\underline{x}-\underline{x}^{\prime}+s \underline{\mu}\right)} e^{-\frac{1}{2}(a(\underline{k})-\imath c(\underline{k})) f_{1}(s, v)}(a(\underline{k})-\imath c(\underline{k})) d \underline{k} \\
& =-2 \frac{f_{2}(s, v)}{\frac{\partial}{\partial v} f_{1}(s, v)} \frac{\imath}{2 \chi} \frac{\partial}{\partial v} \sum_{j=1}^{n} \sigma_{j} \rho_{v, j} \int_{\mathbb{R}^{n}} k_{j} e^{-\imath \underline{k}^{T}\left(\underline{x}-\underline{x}^{\prime}+s \underline{\mu}\right)} e^{-\frac{1}{2}(a(\underline{k})-\imath c(\underline{k})) f_{1}(s, v)} d \underline{k} \\
& =-\frac{f_{2}(s, v)}{\frac{\partial}{\partial v} f_{1}(s, v)} \frac{1}{\chi} \frac{\partial}{\partial v} \sum_{j=1}^{n} \sigma_{j} \rho_{v, j} \frac{\partial}{\partial x_{j}^{\prime}} \int_{\mathbb{R}^{n}} e^{-\imath \underline{k}^{T}\left(\underline{x}-\underline{x}^{\prime}+s \underline{\mu}\right)} e^{-\frac{1}{2}(a(\underline{k})-\imath c(\underline{k})) f_{1}(s, v)} d \underline{k}, \\
& \quad s>0, \underline{k} \in \mathbb{R}^{n}, v>0 .
\end{aligned}
$$

From Eq. (67) we obtain $M_{1}$ :

$$
\begin{gathered}
M_{1}\left(s, \underline{x}, \underline{x}^{\prime}, v\right)=-\frac{1}{\chi} \frac{f_{2}(s, v)}{\frac{\partial}{\partial v} f_{1}(s, v)} \frac{\partial}{\partial s} \sum_{j=1}^{n} \sigma_{j} \rho_{v, j} \frac{\partial}{\partial x_{j}^{\prime}} M_{0}\left(s, \underline{x}, \underline{x}^{\prime}, v\right) \\
=-\frac{f_{2}(s, v)}{\chi \frac{\partial}{\partial v} f_{1}(s, v)} \frac{\partial}{\partial v}\left\{\frac{M_{0}\left(s, \underline{x}, \underline{x}^{\prime}, v\right)}{f_{1}(s, v)} \sum_{j=1}^{n} \sigma_{j} \rho_{v, j}\left[\Gamma^{-1}\left(\underline{x}-\underline{x}^{\prime}+s \underline{\mu}-\frac{\tilde{m}}{2} f_{1}(s, v) \underline{\sigma}\right)\right]_{j}\right\}, \\
s>0, \underline{k} \in \mathbb{R}^{n}, v>0,
\end{gathered}
$$

where $[\cdot]_{j}$ denotes the $j$-th component of the vector $\cdot$

Thanks to formulas (66) and (68), we obtain the approximation of the cumulative distribution function given in (26). 


\section{Appendix C: Explicit formulas for the moments of the yield variables}

Let us deduce the explicit expression for the moments of the variable $x_{j, t}, j=1,2, \ldots, n$, (see Eqs. (1), (2)).

$$
\mathcal{M}_{j, m}(s, \underline{x}, v)=\int_{\mathbb{R}^{n}}\left(x_{j}^{\prime}\right)^{m} M\left(s, \underline{x}, \underline{x}^{\prime}, v\right) d \underline{x}^{\prime},
$$

where $M$ is the marginal conditional density in Eq. (11). By using the Fourier transform of $M$, Eq. (69) becomes:

$$
\begin{aligned}
& \mathcal{M}_{j, m}(s, \underline{x}, v)=\int_{\mathbb{R}^{n}}\left(x_{j}^{\prime}\right)^{m} \frac{1}{(2 \pi)^{n}}\left[\int_{\mathbb{R}^{n}} e^{-\imath \underline{k}^{T}\left(\underline{x}-\underline{x}^{\prime}\right)} \hat{M}(s, \underline{k}, v) d \underline{k}\right] d \underline{x}^{\prime}= \\
& \int_{\mathbb{R}^{n}} d \underline{x}^{\prime}\left(x_{j}^{\prime}-x_{j}+x_{j}\right)^{m} \frac{1}{(2 \pi)^{n}} \int_{\mathbb{R}^{n}} e^{-\imath \underline{k}^{T}\left(\underline{x}-\underline{x}^{\prime}\right)} \hat{M}(s, \underline{k}, v) d \underline{k}= \\
& \sum_{q=0}^{m}\left(\begin{array}{c}
m \\
q
\end{array}\right) x_{j}^{m-q} \int_{\mathbb{R}^{n}} \hat{M}(s, \underline{k}, v) \frac{1}{(2 \pi)^{n}}\left[\int_{\mathbb{R}^{n}} e^{-\imath \underline{k}^{T}\left(\underline{x}-\underline{x}^{\prime}\right)}\left(x_{j}^{\prime}-x_{j}\right)^{q} d \underline{x^{\prime}}\right] d \underline{k}= \\
& \sum_{q=0}^{m}\left(\begin{array}{c}
m \\
q
\end{array}\right) x_{j}^{m-q} \int_{\mathbb{R}^{n}} \hat{M}(s, \underline{k}, v)(-\imath)^{q} \frac{\partial^{q} \delta(\underline{k})}{\partial k_{j}^{q}} d \underline{k} .
\end{aligned}
$$

¿From (70), by using some properties of Dirac's delta function, we obtain:

$$
\begin{aligned}
& \mathcal{M}_{j, m}(s, \underline{x}, v)=\left.\sum_{q=0}^{m}\left(\begin{array}{c}
m \\
q
\end{array}\right) x_{j}^{m-q} \imath^{q} \frac{\partial^{q}}{\partial k_{j}^{q}} \hat{M}(s, \underline{k}, v)\right|_{\underline{k}=\underline{0}} \\
& =\sum_{q=0}^{m}\left(\begin{array}{c}
m \\
q
\end{array}\right) x_{j}^{m-q} \imath^{q} L_{j, q}^{*}(s, v), \quad s>0, \underline{x} \in \mathbb{R}^{n}, v>0 .
\end{aligned}
$$

where $L_{0,0}^{*}(s, v)=\hat{M}(s, 0, v)$ and $L_{j, q}^{*}(s, v)=\left.\frac{\partial^{q}}{\partial k_{j}^{q}} M(s, \underline{k}, v)\right|_{\underline{k}=\underline{0}}, j=1,2, \ldots, n, q=$ $1,2, \ldots$

We prove that $L_{j, q}$ are polynomial functions of $v$ and we give a recursive formula to compute these functions.

By deriving Eqs. (40)-(41) with respect to $k_{j}$ and choosing $\underline{k}=\underline{0}$, we obtain the functions $L_{j, q}, j=0,1, \ldots, n, q=1,2, \ldots$ to be the solution of suitable problems. Specifically,

$L_{0,0}^{*}$ is solution of the equation:

$$
\frac{\partial L_{0,0}^{*}}{\partial s}=\epsilon^{2} \frac{v}{2} \frac{\partial^{2} L_{0,0}^{*}}{\partial v^{2}}+\chi(\theta-v) \frac{\partial L_{0,0}^{*}}{\partial v},
$$

with the initial condition:

$$
L_{0,0}^{*}(0, v)=1
$$


$L_{j, 1}^{*}, j=1,2, \ldots, n$, are solutions of the equations:

$$
\begin{gathered}
\frac{\partial L_{j, 1}^{*}}{\partial s}=\epsilon^{2} \frac{v}{2} \frac{\partial^{2} L_{j, 1}^{*}}{\partial v^{2}}+\chi(\theta-v) \frac{\partial L_{j, 1}^{*}}{\partial v}+\imath\left(\tilde{m} \sigma_{j}^{2} \frac{v}{2}-\mu_{j}\right) L_{0}^{*}-\imath v \epsilon \rho_{v, j} \sigma_{j} \frac{\partial L_{0,0}^{*}}{\partial v}, \\
j=1,2, \ldots, n,
\end{gathered}
$$

with the initial conditions:

$$
\hat{L}_{j, 1}^{*}(0, v)=0, \quad j=1,2, \ldots, n
$$

$L_{j, q}, j=1,2, \ldots, n, q=2,3, \ldots$, are solutions of the equations:

$$
\begin{gathered}
\frac{\partial L_{j, q}^{*}}{\partial s}=\epsilon^{2} \frac{v}{2} \frac{\partial^{2} L_{j, q}^{*}}{\partial v^{2}}+\chi(\theta-v) \frac{\partial L_{j, q}^{*}}{\partial v}-\imath \epsilon v q \sigma_{j} \rho_{j, v} \frac{\partial L_{j, q-1}^{*}}{\partial v} \\
+\imath q\left(\tilde{m} \sigma_{j}^{2} \frac{v}{2}-\mu_{j}\right) L_{j, q-1}^{*}-v \frac{q(q-1)}{2} \sigma_{j}^{2} L_{j, q-2}^{*}, \quad j=1,2, \ldots, n, q=2,3, \ldots,
\end{gathered}
$$

with the initial conditions:

$$
L_{j, q}^{*}(0, v)=0, \quad j=1,2, \ldots, n, \quad q=2,3, \ldots
$$

It is easy to see that the solution of problem (72)-(73) is given by:

$$
L_{0,0}^{*}(s, v)=1
$$

so that, by substituting Eq. (78) into Eqs. (74)-(75), we obtain:

$$
\frac{\partial L_{j, 1}^{*}}{\partial s}=\epsilon^{2} \frac{v}{2} \frac{\partial^{2} L_{j, 1}^{*}}{\partial v^{2}}+\chi(\theta-v) \frac{\partial L_{j, 1}^{*}}{\partial v}+\imath\left(\tilde{m} \sigma_{j}^{2} \frac{v}{2}-\mu_{j}\right)
$$

with initial condition (75).

We look for the solution of Eq. (79) in the form $L_{j, 1}^{*}=f_{j, 1,0}(s)+v f_{j, 1,1}(s)$ and we obtain:

$$
\begin{gathered}
\frac{d}{d s} f_{j, 1,0}=\chi \theta f_{j, 1,1}-\imath \mu_{j} \\
\frac{d}{d s} f_{j, 1,1}+\chi f_{j, 1,1}=\frac{\imath \tilde{m} \sigma_{j}^{2}}{2}
\end{gathered}
$$

with initial conditions:

$$
f_{j, 1, l}(0)=0, l=0,1
$$

An easy computation gives:

$$
\begin{aligned}
f_{j, 1,0}(s) & =\frac{\imath \theta \tilde{m} \sigma_{j}^{2}}{2}\left(s-\psi_{1}(s)\right)-\imath s \mu_{j}, \\
f_{j, 1,1}(s) & =\frac{\imath \theta \tilde{m} \sigma_{j}^{2}}{2} \psi_{1}(s)
\end{aligned}
$$


where $\psi_{1}$ is the function of time defined by Eq. (97). Using Eq. (83), we obtain:

$$
L_{j, 1}^{*}(s, v)=\frac{\imath \tilde{m}}{2} \sigma_{j}^{2}\left[\theta\left(s-\psi_{1}(s)\right)+v \psi_{1}(s)\right]-\imath \mu_{j} s, j=1,2, \ldots, n .
$$

Similarly, we look for the solution of Eq. (76) in the form:

$$
L_{j, q}^{*}(s)=\sum_{d=0}^{q} v^{d} f_{j, q, d}(s), j=1,2, \ldots, n, q=2,3, \ldots .
$$

In order to satisfy the initial conditions $(77)$ the functions $f_{j, q, d}$ must satisfy the following conditions at $s=0$ :

$$
f_{j, q, d}(0)=0
$$

By substituting Eq. (85) into Eq. (76), we obtain the following problems depending on the powers of $v$ :

- power $d=0$ :

$$
\frac{d}{d s} f_{j, q, 0}=\chi \theta f_{j, q, 1}-\imath \mu_{j} q f_{j, q-1,0}
$$

- power $1 \leq d \leq q-1$ :

$$
\begin{aligned}
& \frac{d}{d s} f_{j, q, d}+\chi d f_{j, q, d}=\frac{\varepsilon^{2}}{2} d(d+1) f_{j, q, d+1}+(d+1) \chi \theta f_{j, q, d+1}-\imath \mu_{j} q f_{j, q-1, d} \\
& -\imath \varepsilon q d \sigma_{j} \rho_{v, j} f_{j, q-1, d}+\frac{\imath}{2} \tilde{m} \sigma_{j}^{2} q f_{j, q-1, d-1}-\frac{q(q-1)}{2} \sigma_{j}^{2} f_{j, q-2, d-1},
\end{aligned}
$$

- power $d=q$

$$
\frac{d}{d s} f_{j, q, q}+\chi q f_{j, q, q}=\frac{\imath \tilde{m}}{2} \sigma_{j}^{2} q f_{j, q-1, q-1} .
$$

Note that Eqs. (87)-(89) can be solved by backward recursion. In fact, we have:

$$
\begin{gathered}
f_{j, q, q}(s)=\frac{\imath \tilde{m}}{2} \sigma_{j}^{2} q e^{-\chi s} \int_{0}^{s} e^{\chi \tau} f_{j, q-1, q-1}(\tau) d \tau \\
f_{j, q, d}(s)=e^{-\chi s} \int_{0}^{s} e^{\chi \tau}\left\{\frac{\varepsilon^{2}}{2} d(d+1) f_{j, q, d+1}(\tau)+(d+1) \chi \theta f_{j, q, d+1}(\tau)-\imath \mu_{j} q f_{j, q-1, d}(\tau)+\right. \\
\left.\frac{\imath}{2} \tilde{m} \sigma_{j}^{2} q f_{j, q-1, d-1}(\tau)-\imath \varepsilon q d \sigma_{j} \rho_{v, j} f_{j, q-1, d}(\tau)-\frac{q(q-1)}{2} \sigma_{j}^{2} f_{j, q-2, d-1}(\tau)\right\} d \tau
\end{gathered}
$$

and

$$
f_{j, q, 0}(s)=\int_{0}^{s}\left[\chi \theta f_{j, q, 1}(\tau)-\imath q f_{j, q-1,0}(\tau)\right] d \tau
$$


The integrals appearing in Eqs. (90), (91) and (92) are elementary integrals that can be computed explicitly.

In fact, by using formulas (90), (91) and (92), we deduce the first four conditional moments of the yield/log-yield variable given that $\underline{x}_{0}=\underline{x}$ and $v_{0}=v$ when $\tilde{m}=0$ and $\mu_{j}=0, j=1,2, \ldots, n$ (i.e. the model used in the numerical experiment in the Eurozone). For $j=1,2, \ldots, n$, they are:

$$
\begin{gathered}
\mathcal{M}_{j, 1}(s, \underline{x}, v)=x_{j} \\
\mathcal{M}_{j, 2}(s, \underline{x}, v)=x_{j}^{2}+\sigma_{j}^{2} f_{1}(s, v) \\
\mathcal{M}_{j, 3}(s, \underline{x}, v)=x_{j}^{3}+3 x_{j} \sigma_{j}^{2} f_{1}(s, v)+3 \varepsilon \sigma_{j}^{3} \rho_{v, j}\left[\frac{1}{\chi} f_{2}(s, v)-s(\theta-v) \psi_{1}(s)\right] \\
\mathcal{M}_{j, 4}(s, \underline{x}, v)=x_{j}^{4}+6 x_{j}^{2} \sigma_{j}^{2} f_{1}(s, v)+12 x_{j} \varepsilon \sigma_{j}^{3} \rho_{v, j}\left[\frac{1}{\chi} f_{2}(s, v)-s(\theta-v) \psi_{1}(s)\right]+L_{j, 4}^{*}(s, v),
\end{gathered}
$$

where $\psi_{m}, m=1,2$ and $L_{j, 4}^{*}$ are given by:

$$
\begin{gathered}
\psi_{m}(s)=\frac{1-e^{-m \chi s}}{m \chi}, m=1,2 \\
L_{j, 4}^{*}(s, v)=6 \frac{v^{2}}{\chi}\left(\psi_{1}(s)-\psi_{2}(s)\right)+6 v \frac{\sigma_{j}^{4}}{\chi^{2}}\left(\epsilon^{2}+2 \chi \theta\right)\left(\psi_{2}(s)-s e^{-\chi s}\right) \\
+6 v \frac{\sigma_{j}^{4} \theta}{\chi}\left(s+s e^{-\chi s}-2 \psi_{1}(s)\right)+12 v \frac{\sigma_{j}^{4} \epsilon^{2}}{\chi^{2}} \rho_{v, j}^{2}\left(\psi_{1}(s)-s e^{-\chi s}-\frac{s^{2}}{2} \chi e^{-\chi s}\right) \\
6 \frac{\sigma_{j}^{4} \theta}{\chi^{2}}\left(\epsilon^{2}+2 \chi \theta\right)\left(\frac{1}{2} s-\frac{1}{2} \psi_{2}(s)+s e^{-\chi s}-\psi_{1}(s)\right)+6 \frac{\sigma_{j}^{4} \theta^{2}}{\chi}\left(\frac{\chi}{2} s^{2}-2 s-s e^{-\chi s}+3 \psi_{1}(s)\right) \\
+12 \frac{\sigma_{j}^{4} \epsilon^{2} \theta}{\chi^{2}} \rho_{v, j}^{2}\left(s-3 \psi_{1}(s)+2 s e^{-\chi s}+\frac{s^{2}}{2} \chi e^{-\chi s}\right) .
\end{gathered}
$$

Using formulas (93)-(96) we obtain:

$$
\begin{gathered}
E\left(\left(x_{j}^{\prime}-x_{0, j}\right)^{2}\right)=\sigma_{j}^{2} f_{1}\left(s, v_{0}\right), \\
E\left(\left(x_{j}^{\prime}-x_{0, j}\right)^{3}\right)=3 \varepsilon \sigma_{j}^{3} \rho_{v_{0}, j}\left[\frac{1}{\chi} f_{2}\left(s, v_{0}\right)-s\left(\theta-v_{0}\right) \psi_{1}(s)\right],
\end{gathered}
$$




$$
\begin{aligned}
& E\left(\left(x_{j}^{\prime}-x_{0, j}\right)^{4}\right)=6 \frac{v_{0}^{2}}{\chi}\left(\psi_{1}(s)-\psi_{2}(s)\right)+6 v_{0} \frac{\sigma_{j}^{4}}{\chi^{2}}\left(\epsilon^{2}+2 \chi \theta\right)\left(\psi_{2}(s)-s e^{-\chi s}\right) \\
& +6 v_{0} \frac{\sigma_{j}^{4} \theta}{\chi}\left(s+s e^{-\chi s}-2 \psi_{1}(s)\right)+12 v_{0} \frac{\sigma_{j}^{4} \epsilon^{2}}{\chi^{2}} \rho_{v, j}^{2}\left(\psi_{1}(s)-s e^{-\chi s}-\frac{s^{2}}{2} \chi e^{-\chi s}\right) \\
& 6 \frac{\sigma_{j}^{4} \theta}{\chi^{2}}\left(\epsilon^{2}+2 \chi \theta\right)\left(\frac{1}{2} s-\frac{1}{2} \psi_{2}(s)+s e^{-\chi s}-\psi_{1}(s)\right)+6 \frac{\sigma_{j}^{4} \theta^{2}}{\chi}\left(\frac{\chi}{2} s^{2}-2 s-s e^{-\chi s}+3 \psi_{1}(s)\right) \\
& +12 \frac{\sigma_{j}^{4} \epsilon^{2} \theta}{\chi^{2}} \rho_{v, j}^{2}\left(s-3 \psi_{1}(s)+2 s e^{-\chi s}+\frac{s^{2}}{2} \chi e^{-\chi s}\right)
\end{aligned}
$$

When $\tilde{m} \neq 0$ and $\mu_{j} \neq 0, j=1,2, \ldots, n$ the first two conditional moments are given by:

$$
\mathcal{M}_{j, 1}(s, \underline{x}, v)=x_{j}+\mu_{j} s-\frac{\tilde{m}}{2} \sigma_{j}^{2}\left(\theta s+(v-\theta) \psi_{1}(s)\right)=x_{j}+\mu_{j} s-\frac{\tilde{m}}{2} \sigma_{j}^{2} f_{1}(s, v),
$$

and

$$
\mathcal{M}_{j, 2}(s, \underline{x}, v)=x_{j}^{2}+2 x_{j}\left(\mathcal{M}_{j, 1}(s, \underline{x}, v)-x_{j}\right)-L_{2}^{*}(s, v)
$$

where $L_{j, 2}^{*}(s, v)=f_{j, 2,2}(s) v^{2}+f_{j, 2,1}(s) v+f_{j, 2,0}(s)$ where $f_{j, 2, m}, m=0,1,2$ are given by:

$$
\begin{gathered}
f_{j, 2,2}(s)=-\frac{\tilde{m}}{4} \sigma_{j}^{4}\left(\psi_{1}(s)\right)^{2} \\
f_{j, 2,1}(s)=-\sigma_{j}^{2} \psi_{1}(s)-\frac{\tilde{m}^{2}}{4}\left(\epsilon^{2}+2 \chi \theta\right) \frac{\sigma_{j}^{4}}{\chi^{2}}\left(2 \psi_{1}(s)-2 s e^{-\chi s}-\chi\left(\psi_{1}(s)\right)^{2}\right. \\
+\tilde{m} \sigma_{j}^{2}\left(\epsilon \sigma_{j} \rho_{v, j}+\mu_{j}\right) \frac{1}{\chi}\left(\psi_{1}(s)-s e^{-\chi s}\right)-\frac{\tilde{m}}{\chi} \sigma_{j}^{2}\left(\frac{\tilde{m}}{2} \sigma_{j}^{2} \theta-\mu_{j}\right)\left(s-\psi_{1}(s)\right) \\
+\frac{\tilde{m}}{2} \sigma_{j}^{4} \frac{\theta}{\chi}\left(\psi_{1}(s)-s e^{-\chi s}\right) \\
f_{j, 2,0}(s)=-\sigma_{j}^{2} \theta\left(s-\psi_{1}(s)\right)-\mu_{j}^{2} s^{2}+\tilde{m} \mu_{j} \sigma_{j}^{2} \theta \frac{s^{2}}{2}-\tilde{m} \mu_{j} \sigma_{j}^{2} \frac{\theta}{\chi}\left(s-\psi_{1}(s)\right) \\
-\frac{\tilde{m}^{2}}{4}\left(\epsilon^{2}+2 \chi \theta\right) \frac{\sigma_{j}^{4} \theta}{\chi^{2}}\left[2 s\left(1+e^{-\chi s}\right)-3 \psi_{1}(s)-s+\frac{1}{2}\left(\psi_{1}(s)\right)^{2}\right] \\
+\tilde{m} \sigma_{j}^{2}\left(\epsilon \sigma_{j} \rho_{v, j}+\mu_{j}\right) \frac{\theta}{\chi}\left(-2 \psi_{1}(s)+s\left(1+e^{-\chi s}\right)\right)-\frac{\tilde{m}}{\chi} \sigma_{j}^{2}\left(\frac{\tilde{m}}{2} \sigma_{j}^{2} \theta-\mu_{j}\right) \theta\left(\frac{s^{2} \chi}{2}-s+\psi_{1}(s)\right) \\
+\frac{\tilde{m}}{2} \sigma_{j}^{4} \frac{\theta^{2}}{\chi}\left(-2 \psi_{1}(s)+s\left(1+e^{-\chi s}\right)\right) .
\end{gathered}
$$

Formulas (102) and (103) allow us to get elementary expressions for variance. 


\section{References}

[1] Afonso, A., Martins, M. M. (2012). Level, slope, curvature of the sovereign yield curve, and fiscal behaviour. Journal of Banking \& Finance, 36(6); pp 1789-1807.

[2] Afonso, A., Strauch, R. (2007). Fiscal Policy Events and Interest Rate Swap Spreads: some Evidence from the EU. Journal of International Financial Markets, Institutions E Money, 17 (3); pp 261-276.

[3] Andersen, T.G., Benzoni, L. (2010). Do bonds span volatility risk in the U.S. treasury market? A specification test for affine term structure models. The Journal of Finance, 65 (2); pp 603-653.

[4] Attinasi, M.G, Checherita, C., and Nickel, C. (2011). What Explains the Surge in Euro Area Sovereign Spreads during the Financial Crisis of 2007-09?. In Kolb, R. W., editor, Sovereign Debt: From Safety to Default; pp 407-414. John Wiley \& Sons, Inc.

[5] Barrios, S., Iversen, P., Lewandowska, M., Setzer, R. (2009). Determinants of intraeuro area government bond spreads during the financial crisis. European Economy Economic Papers, 388, November.

[6] Baum, C. F., Caglayan, M., Stephan, A., Talavera, O. (2004). Uncertainty determinants of corporate liquidity. Economic Modelling, 25(5); pp 833-849.

[7] Borio, C., Disyatat, P. (2010). Unconventional monetary policies: an appraisal. The Manchester School, 78(s1); pp 53-89.

[8] Chen, Y., Tsang, K.P. (2013) What Does the Yield Curve Tell Us About Exchange Rate Predictability? Review of Economics and Statistics 95, (1); pp. 185-205.

[9] Cheredito, P., Filipovic, D., Kimmel, R. (2007) Market Price of Risk Specifications for Affine Models: Theory and Evidence. Journal of Financial Economics 83, (1); pp 123-170.

[10] Chiarella, C., Kwon, O. K. (2003). Finite dimensional affine realisations of HJM models in terms of forward rates and yields. Review of Derivatives Research, 6(2); pp 129-155.

[11] Christensen, J. H. E., Diebold, F. X., Rudebush, G.D. (2011). The affine arbitragefree class of Nelson-Siegel term structure models. Journal of Econometrics 164 (1); pp 4-20. 
[12] Christensen, J. H. E., Lopez, J. A., Rudebush, G.D. (2014). Can spanned term structure factors drive stochastic yield volatility? Federal Reserve Bank of San Francisco Working Paper Series 2014-03.

[13] Collin-Dufresne, P., Goldstein, R.S. Jones, C.S.(2009) Can interest rate volatility be extracted from the cross-section of bond yields? Journal of Financial Economics 94 (1); pp 47-66.

[14] Coroneo, L., Nyholm, K., Vidova-Koleva, R. (2011). How arbitrage-free is the NelsonSiegel model?. Journal of Empirical Finance, 18(3); pp 393-407.

[15] Dai, Q., Singleton, K. (2002). Expectation puzzles, time-varying risk premia, and affine models of the term structure. Journal of Financial Economics 63 (3); pp 415441.

[16] Duffie, D., Pan, J. Singleton, K. (2000) Transform analysis and asset pricing for affine jump-Diffusions. Econometrica, 68; pp. 1343-1376.

[17] Diebold F.X., Li, C. (2006). Forecasting the term structure of government bond yields. Journal of Econometrics, 130; pp 337-364.

[18] Diebold, F. X., Rudebusch, G. D., Aruoba, S. B. (2006b). The macroeconomy and the yield curve: a dynamic latent factor approach. Journal of econometrics, 131(1); pp 309-338.

[19] Diebold, F. X., Li, C., Yue, V. Z. (2008). Global yield curve dynamics and interactions: a dynamic NelsonSiegel approach. Journal of Econometrics, 146(2); pp 351-363.

[20] Diebold, F. X., Rudebusch, G. D. (2013). Yield Curve Modeling and Forecasting: The Dynamic Nelson-Siegel Approach. Princeton University Press.

[21] Dong-Hyun, A., Dittmar, F., Gallant, A.R. (2002). Quadratic term structure models: theory and evidence. Review of Financial Studies, 15(1); pp 243-288.

[22] Duffee, G. (2002). Term premia and interest rate forecasts in affine models. Journal of Finance 57 (1); pp 405-443.

[23] Duffie, D., Pan, J., Singleton, K. (2000) Transform analysis and asset pricing for Affine jump-diffusions, Econometrica 68(6); pp 1343-1376.

[24] Ehrmann, M., Fratzscher, M., Gurkaynak, R.S., Swanson, E.T. (2011). Convergence and anchoring of yield curves in the Euro area. The Review of Economics and Statistics, 93 (1); pp 350-364. 
[25] Erber, G. (2011). Italy's fiscal crisis. Intereconomics 46 (6); pp 332-339.

[26] Eser, F., Carmona Amaro, M., Iacobelli, S., Rubens, M. (2012). The use of the Eurosystem's monetary policy instruments and operational framework since 2009. ECB occasional paper, (135).

[27] Ghosal, V., Loungani, P. (2000). The differential impact of uncertainty on investment in small and large business. The Review of Economics and Statistics, 82 (2); pp 338-349.

[28] Giannone, D., Lenza, M., Pill, H., Reichlin, L. (2011) Non-standard monetary policy measures and monetary developments. ECB Working Paper Series 1290, January 2011.

[29] Grilli, R., Tedeschi, G., Gallegati, M. (2014a) Bank interlinkages and Macroeconomic stability. International Review of Economics and Finance 34; pp 72-88.

[30] Grilli, R., Tedeschi, G., Gallegati, M. (2014b) Markets connectivity and financial contagion. Journal of Economic Interaction and Coordination, DOI10.1007/s11403$014-0129-1$.

[31] Haugh, D., Ollivaud, P., Turner, D. (2009) What Drives Sovereign Risk Premiums. OECD Economics Department Working Papers 718.

[32] Hautsch, N., Ou, Y. (2012) Analyzing interest rate risk: stochastic volatility in the term structure of government bond yields. Journal of Banking and Finance 36 (11); pp 2988-3007.

[33] Heston, S. L. (1993). A closed-form solution for options with stochastic volatility with applications to bond and currency options. Review of financial studies, 6(2); pp 327-343.

[34] Homescu, C. (2011). Implied volatility surface: construction methodologies and characteristics. SSRN library, 2011: http://arxiv.org/pdf/1107.1834.pdf.

[35] Lucas, A., Schwaabb, B., Zhang, X. (2014). Conditional Euro Area Sovereign Default Risk. Journal of Business and Economic Statistics 32 (2); pp 271-284.

[36] Mancino, M.E., Recchioni, M.C. (2015). Fourier Spot Volatility Estimator: Asymptotic Normality and Efficiency with Liquid and Illiquid High-Frequency Data. PLoS ONE 10(9), e0139041. doi : 10.1371/journal.pone.0139041

[37] Manganelli, S., Wolswijk, G. (2009). What drives spreads in the euro area government bond market?. Economic Policy, 24 (58); pp 191-240. 
[38] Mesters, G., Schwaabb, B., Koopman, S.J. (2014). A dynamic yield curve model with stochastic volatility and non-Gaussian interactions: an empirical study of nonstandard monetary policy in the euro area. Tinbergen Institute Discussion Paper 14-071/III. Available at SSRN: http://ssrn.com/abstract=2456624.

[39] Miglierina, E., Molho, E., Recchioni, M.C. (2008). Box-constrained multi-objective optimization: a gradient-like method without "a priori" scalarization. European Journal of Operational Research 188; pp 662-682.

[40] Nelson, C., Siegel, A. (1987). Parsimonious modeling of yield curves. Journal of Business, 60; pp 473-489.

[41] Nickel, C., Rother, P., Ruelke, J. C. (2011). Fiscal variables and bond spreadsevidence from Eastern European countries and Turkey. Applied Financial Economics, 21(17); pp 1291-1307.

[42] Nielsen, M.O., Frederiksen, P. (2007). Finite sample accuracy and choice of sampling frequency in integrated volatility estimation. Journal of Empirical Finance 15 (2); pp 265-286.

[43] Pacelli, G., Recchioni, M.C. (2000). A monotonic variable metric algorithm for linearly constrained nonlinear programming. Journal of Optimization Theory and Applications 104; pp 255-279.

[44] Poon, S-H., Granger, W.J. (2003). Forecasting volatility in financial markets: a review. Journal of Economic Literature, 41(2), pp 478-539.

[45] Rault, C., Afonso, A. (2011). Long-run Determinants of Sovereign Yields. Economics Bulletin, 31 (1), pp 367-374.

[46] Recchioni, M.C., Tedeschi, G., Gallegati, M. (2015). A calibration procedure for analyzing stock price dynamics in an Agent-based framework. Journal of Economic Dynamic and control 60; pp 1-25.

[47] Sgherri, S., Zoli, E., (2009). Euro Area Sovereign Risk During the Crisis. International Monetary Fund Working Paper No. 09/222; pp 1-23.

[48] Trolle, A.B., Schwartz, E.S. (2009). A general stochastic volatility model for the pricing of interest rate derivatives. Review of Financial Studies, 22 (5); pp 20072057.

[49] Von Hagen, J., Schuknecht, L., Wolswijk, G. (2011). Government bond risk premiums in the EU revisited: The impact of the financial crisis. European Journal of Political Economy, 27(1); pp 36-43. 